\title{
Exponential behavior and upper noise excitation index of solutions to evolution equations with unbounded delay and tempered fractional
} Brownian motions

\author{
Yejuan Wang · Yarong Liu · Tomás Caraballo
}

Received: date / Accepted: date

\begin{abstract}
In this paper we investigate stochastic evolution equations with unbounded delay in fractional power spaces perturbed by a tempered fractional Brownian motion $B_{Q}^{\sigma, \lambda}(t)$ with $-1 / 2<\sigma<0$ and $\lambda>0$. We first introduce a technical lemma which is crucial in our stability analysis. Then we prove the existence and uniqueness of mild solutions by using semigroup methods. The upper nonlinear noise excitation index of the energy solutions at any finite time $t$ is also obtained. Finally, we consider the exponential asymptotic behavior of mild solutions in mean square.
\end{abstract}

Keywords Stochastic PDEs · Unbounded delay · Tempered fractional Brownian motion · Fractional powers of closed operators $\cdot$ Exponential decay in mean square

\section{Introduction}

Tempered fractional Brownian motion (TFBM) defined by exponentially tempering the power law kernel in the moving average representation of a fractional Bownian motion (FBM) was first introduced by

This work was supported by NSF of China (Grant No. 41875084), the Fundamental Research Funds for the Central Universities under Grant Nos. lzujbky-2018-ot03 and lzujbky-2018-it58. The research of T. Caraballo has been partially supported by Ministerio de Ciencia Innovación y Universidades (Spain), FEDER (European Community) under grant PGC2018-096540B-I00, and by Junta de Andalucía (Consejería de Economía y Conocimiento) and FEDER under projects US-1254251 and P18-FR-4509.

Yejuan Wang · Yarong Liu

School of Mathematics and Statistics,

Gansu Key Laboratory of Applied Mathematics and Complex Systems,

Lanzhou University, Lanzhou 730000, PR China

E-mail: wangyj@lzu.edu.cn, liuyr19@lzu.cn

T. Caraballo

Departamento de Ecuaciones Diferenciales y Análisis Numérico,

Facultad de Matemáticas, Universidad de Sevilla,

c/ Tarfia s/n, 41012-Sevilla, Spain

E-mail: caraball@us.es 
Meerschaert and Sabzikar in [27]. Tempered fractional Gaussian noise (TFGN), the increments of TFBM, can exhibit semi-long range dependence when the corresponding FGN is long range dependent. Wind speed data are important for electrical power generation and structural engineering. An important application to model wind speed near the earth surface was also presented in [27]. More precisely, TFGN can provide a useful stochastic process model for wind speed data, see, e.g., [3, 10, 17, 23, 29]. Furthermore, the time-changed TFBM has been investigated in [9] with potential applications in financial time series, biology and physics.

Retarded differential equations have attracted much attention in the literature due to physical reasons with non-instant transmission phenomena such as high velocity fields in wind tunnel experiments, or other memory processes, or biological motivations like species growth or incubating time in disease models among many others. Stochastic delay differential equations driven by the standard Brownian motion have been widely investigated in the literature, see, e.g., $[7,8,21,26,32,33]$ and the references therein. There has, however, been little mention of SDEs or SPDEs with delay driven by TFBM. In this paper we consider the stochastic evolution equations with infinite delay

$$
\left\{\begin{array}{l}
d u(t)=-A u(t) d t+f\left(t, u_{t}\right) d t+g\left(t, u_{t}\right) d B_{Q}^{\sigma, \lambda}(t), \quad t>0 \\
u(t)=\varphi(t), \quad t \in(-\infty, 0]
\end{array}\right.
$$

where $-A$ is a closed, densely defined linear operator generating an analytic semigroup $S(t), t \geq 0$, on a separable Hilbert space $\mathcal{H}, f:[0, \infty) \times \mathscr{C}\left(\mathcal{H}^{\alpha}\right) \mapsto \mathcal{H}, g:[0, \infty) \times \mathscr{C}\left(\mathcal{H}^{\alpha}\right) \mapsto L_{Q}^{0}(\mathcal{U}, \mathcal{H})$ are two Lipschitz continuous functions, $B_{Q}^{\sigma, \lambda}(t)$ is a tempered fractional Brownian motion with $-1 / 2<\sigma<0$ and $\lambda>0$ over a filtered probability space $\left(\Omega, \mathcal{F},\left(\mathcal{F}_{t}\right)_{t \geq 0}, P\right), \varphi \in \mathscr{C}\left(\mathcal{H}^{\alpha}\right)$ with $\varphi(t)$ being $\mathcal{F}_{t}$-measurable, where $\mathcal{F}_{t}=\mathcal{F}_{0}$ for all $t \leq 0$. Here $\mathcal{H}^{\alpha}=D\left(A^{\alpha}\right)$ and

$$
\mathscr{C}\left(\mathcal{H}^{\alpha}\right)=\left\{\psi \in C\left(-\infty, 0 ; L^{2}\left(\Omega ; \mathcal{H}^{\alpha}\right)\right): \lim _{\theta \rightarrow-\infty} \psi(\theta) \text { exists in } L^{2}\left(\Omega ; \mathcal{H}^{\alpha}\right)\right\}
$$

In $[15,16]$, the existence of a unique pathwise solution for stochastic evolution equations driven by FBM was established when $H \in(1 / 3,1 / 2]$. In $[13,14]$, the existence and uniqueness of solutions for delayed SDEs driven by FBM have been proved when $H>1 / 2$. Using rough path theory, the authors gave the existence and uniqueness of solutions to fractional equations with delay when $H>1 / 3$ (see, e.g., [30]). In [6,18], the authors investigated the existence, uniqueness and exponential asymptotic behavior of mild solutions to stochastic delay equations perturbed by FBM with $H>1 / 2$. Controllability of non-autonomous neutral evolution stochastic functional differential equations driven by $\mathrm{FBM}$ with $H>1 / 2$ has been proved in [24]. More recently, the global existence, uniqueness and viability results to stochastic functional differential equations in Hilbert spaces driven by FBM when $H>1 / 2$ have been studied in [34]. However, the literature about SDEs or SPDEs driven by TFBM is scarce in both cases with and without delay.

The purpose of this paper is to investigate the global existence and uniqueness of mild solutions to stochastic delay evolution equations (1.1) in fractional power spaces, and to study the effect of nonlinear noise to (1.1) but with $f=0$ when the noise is large, and also to analyze the long time behavior to (1.1) but in the particular case in which the function $g$ becomes independent of the state variable, in other words, 
when $g$ is replaced by $\phi:[0, \infty) \mapsto \mathcal{L}_{Q}^{0}(\mathcal{U}, \mathcal{H})$. The reason to consider this particular situation is explained in details in Section 5. "Intermittency" is the property that the solution $u_{t}(x)$ develops extreme oscillations at some values of $x$, typically when $t$ is large. Intermittency has been observed in an enormous number of scientific disciplines such as "spikes" in neural activity or "shocks" in finance among many others. It is worth noticing that, in NMR spectroscopy, intermittency can be strongly associated to nonlinear noise excitation (see, e.g., [1,22]). The effect of noise intensity on stochastic parabolic equations driven by Brownian motion has been discussed in recent years, in particular the relationship between the energy of solutions at time $t$ and the level of the noise was established in $[12,25,19,20]$. However, there has been little literature about the relationship between the energy of solutions and the level of the noise for stochastic delay evolution equations even in the case of Brownian motion. Here we consider stochastic evolution equations with infinite delay and TFBM, the upper bound of the upper excitation index of the solution at time $t$ will be presented. $\underline{e}(t)$ and $\bar{e}(t)$, respectively, denote the lower and upper excitation indices of the mild solution at time $t[12$, $25,19,20]$, where we may use the notation

$$
\underline{e}(t):=\liminf _{\eta \rightarrow \infty} \frac{\log \log \mathscr{E}_{t}(\eta)}{\log \eta}, \quad \bar{e}(t):=\limsup _{\eta \rightarrow \infty} \frac{\log \log \mathscr{E}_{t}(\eta)}{\log \eta}
$$

where $\mathscr{E}_{t}$ stands for the energy of the solution at time $t$ and $\eta$ stands for the level of the noise.

The contents of the paper are as follows. In Section 2 some necessary preliminaries on the stochastic integration with respect to TFBM are established. In particular, a technical lemma which is crucial in our analysis is proved. In Section 3 the global existence and uniqueness of mild solutions to (1.1) are established. In Section 4 we show an upper bound of the upper excitation index of the mild solution to (1.1) at time $t$ but with $f=0$. The last section is devoted to establishing some sufficient conditions ensuring the exponential decay to zero of the mild solution to (1.1) in mean square, but in the particular case in which $g$ possesses the form $g\left(t, u_{t}\right)=\phi(t)$, with $\phi:[0, \infty) \mapsto \mathcal{L}_{Q}^{0}(\mathcal{U}, \mathcal{H})$.

\section{Preliminaries}

In this section, we introduce the tempered fractional Brownian motion as well as the Wiener integral with respect to it; for more details, we refer to $[27,28]$. We also establish some important results which will be used throughout the paper.

We denote by $\mathcal{H}$ a separable Hilbert space with inner product $(\cdot, \cdot)$ and norm $\|\cdot\|$. Let $\mathcal{U}$ be another separable Hilbert space and $\mathcal{L}(\mathcal{U}, \mathcal{H})$ be the space of all bounded linear operators from $\mathcal{U}$ into $\mathcal{H}$. For convenience, we will use the same notation $\|\cdot\|$ to denote the norms in $\mathcal{U}$ and $\mathcal{L}(\mathcal{U}, \mathcal{H})$, and use $(\cdot, \cdot)$ to denote the inner product of $\mathcal{U}$ without any confusion. Let $(\Omega, \mathcal{F}, P)$ be a probability space on which an increasing and right continuous family $\left\{\mathcal{F}_{t}\right\}_{t \geq 0}$ of complete sub- $\sigma$-algebras of $\mathcal{F}$ is defined, and $\mathcal{F}_{0}$ contains all $P$-null sets of $\mathcal{F}$.

Now let us recall the definition and some basic properties of tempered fractional Brownian motion (TFBM). Let $\{B(t)\}_{t \in \mathbb{R}}$ be a two-sided one-dimensional Brownian motion with mean zero and variance 
$|t|$ for all $t \in \mathbb{R}$. Define an independently scattered Gaussian random measure $B(d x)$ with control measure $m(d x)=d x$ by setting $B[a, b]=B(b)-B(a)$ for any real numbers $a<b$, and then extending to all Borel sets.

Definition 1 For any $\sigma<1 / 2$ and $\lambda>0$, a tempered fractional Brownian motion (TFBM) is defined by the following integral:

$$
B^{\sigma, \lambda}(t)=\int_{-\infty}^{\infty}\left[e^{-\lambda(t-x)_{+}}(t-x)_{+}^{-\sigma}-e^{-\lambda(-x)_{+}}(-x)_{+}^{-\sigma}\right] B(d x),
$$

where $(x)_{+}=x I_{(x>0)}, 0^{0}=0$ and $\lambda$ is called tempered parameter.

It follows from Proposition 2.3 in [27] that TFBM has the covariance function

$$
\operatorname{Cov}\left[B^{\sigma, \lambda}(t), B^{\sigma, \lambda}(s)\right]=\frac{1}{2}\left[C_{t}^{2}|t|^{2 H}+C_{s}^{2}|s|^{2 H}-C_{t-s}^{2}|t-s|^{2 H}\right],
$$

where $H=1 / 2-\sigma$, and

$$
C_{t}^{2}=\frac{2 \Gamma(2 H)}{(2 \lambda|t|)^{2 H}}-\frac{2 \Gamma\left(H+\frac{1}{2}\right)}{\sqrt{\pi}} \frac{1}{(2 \lambda|t|)^{H}} K_{H}(\lambda|t|), \quad t \neq 0
$$

in which $K_{H}(\cdot)$ is the modified Bessel function of the second kind, and $C_{0}^{2}=0$.

When $\lambda=0$ and $-1 / 2<\sigma<1 / 2$, the TFBM (2.1) reduces to a fractional Brownian motion (FBM), a self-similar Gaussian stochastic process with Hurst scaling index $H=1 / 2-\sigma$. When $\lambda=0$ and $\sigma<-1 / 2$, TFBM (2.1) does not exist, since the integrand in the right hand of (2.1) is not in $L^{2}(\mathbb{R})$. However, TFBM with $\lambda>0$ and $\sigma<-1 / 2$ is well-defined, because the exponential tempering keeps the integrand in $L^{2}(\mathbb{R})$. When $\sigma<-1 / 2$ and $\lambda>0$, or when $\sigma=0$ and $\lambda>0$, TFBM (2.1) is a continuous semimartingale, so the classical Itô stochastic calculus is applicable to TFBM in these cases. When $\sigma \in(-1 / 2,0) \cup(0,1 / 2)$ and $\lambda>0$, TFBM is neither a semimartingale nor a Markov process.

We assume that there exists a complete orthonormal basis $\left\{e_{k}\right\}_{k \in \mathbb{N}}$ in $\mathcal{U}$, and that $B_{Q}^{\sigma, \lambda}=\left\{B_{Q}^{\sigma, \lambda}(t)\right\}_{t \geq 0}$, $B_{Q}^{H}=\left\{B_{Q}^{H}(t)\right\}_{t \geq 0}$ and $B_{Q}=\left\{B_{Q}(t)\right\}_{t \geq 0}$, respectively, are cylindrical $\mathcal{U}$-valued TFBM, FBM and Brownian motion defined on $\left(\Omega, \mathcal{F},\left\{\mathcal{F}_{t}\right\}_{t \geq 0}, P\right)$ with a finite trace nuclear covariance operator $Q \geq 0$. Denote $\operatorname{Tr}(Q)=$ $\Sigma_{k=1}^{\infty} \lambda_{k}<\infty$, which satisfies that $Q e_{k}=\lambda_{k} e_{k}, k \in \mathbb{N}$. Let $\left\{B_{k}^{\sigma, \lambda}\right\}_{k \geq 1}$ be a sequence of two-sided onedimensional TFBMs mutually independent on $\left(\Omega, \mathcal{F},\left\{\mathcal{F}_{t}\right\}_{t \geq 0}, P\right)$ such that

$$
B_{Q}^{\sigma, \lambda}(t)=\sum_{k=1}^{\infty} \sqrt{\lambda_{k}} B_{k}^{\sigma, \lambda}(t) e_{k}, \quad t \geq 0,
$$

where $-1 / 2<\sigma<0$ and $\lambda>0$. In particular, let $\left\{B_{k}^{H}\right\}_{k \geq 1}$ and $\left\{B_{k}\right\}_{k \geq 1}$, respectively, be the sequences of two-sided one-dimensional standard FBMs and Brownian motions mutually independent on $\left(\Omega, \mathcal{F},\left\{\mathcal{F}_{t}\right\}_{t \geq 0}, P\right)$ such that

$$
B_{Q}^{H}(t)=\sum_{k=1}^{\infty} \sqrt{\lambda_{k}} B_{k}^{H}(t) e_{k}, \quad t \geq 0
$$


and

$$
B_{Q}(t)=\sum_{k=1}^{\infty} \sqrt{\lambda_{k}} B_{k}(t) e_{k}, \quad t \geq 0,
$$

where Hurst index $H \in(1 / 2,1)$.

For $\psi, \phi \in \mathcal{L}(\mathcal{U}, \mathcal{H})$, we define $(\psi, \phi)_{Q}=\operatorname{Tr}\left(\psi Q \phi^{*}\right)$, where $\phi^{*}$ is the adjoint of the operator $\phi$. Then, for any bounded operator $\phi \in \mathcal{L}(\mathcal{U}, \mathcal{H})$,

$$
\|\phi\|_{Q}^{2}=\operatorname{Tr}\left(\phi Q \phi^{*}\right)=\sum_{k=1}^{\infty}\left\|\sqrt{\lambda_{k}} \phi e_{k}\right\|^{2} .
$$

If $\|\phi\|_{Q}^{2}<\infty$, then $\phi$ is called a $Q$-Hilbert-Schmidt operator. Denote by $\mathcal{L}_{Q}^{0}(\mathcal{U}, \mathcal{H})$ the space of all $\phi \in \mathcal{L}(\mathcal{U}, \mathcal{H})$ such that $\phi$ is a $Q$-Hilbert-Schmidt operator equipped with the norm $\|\cdot\|_{Q}$.

Now we recall the definitions of tempered fractional integral and stochastic integral with respect to TFBM; see [28].

Definition 2 For any $f \in L^{p}(0, T)$ (where $\left.1 \leq p<\infty\right)$, and for any $a, b \in[0, T]$ with $b>a$, the positive and negative tempered fractional integral on $(a, b)$ are defined by

$$
\mathbb{I}_{a+}^{\alpha, \lambda} f(t)=\frac{1}{\Gamma(\alpha)} \int_{a}^{t} f(u)(t-u)^{\alpha-1} e^{-\lambda(t-u)} d u
$$

and

$$
\mathbb{I}_{b-\lambda}^{\alpha, \lambda} f(t)=\frac{1}{\Gamma(\alpha)} \int_{t}^{b} f(u)(u-t)^{\alpha-1} e^{-\lambda(u-t)} d u
$$

respectively, for any $\alpha>0$ and $\lambda>0$, where $\Gamma(\alpha)=\int_{0}^{+\infty} e^{-x} x^{\alpha-1} d x$ is the Euler gamma function.

Definition 3 For any $-1 / 2<\sigma<0, \lambda>0$, and for any $a, b \in[0, T]$ with $b>a$, we define

$$
\int_{a}^{b} f(t) d B^{\sigma, \lambda}(t):=\Gamma(k+1) \int_{a}^{b}\left(\mathbb{I}_{b-}^{k, \lambda} f(t)-\lambda \mathbb{I}_{b-}^{k+1, \lambda} f(t)\right) d B(t)
$$

for any $f \in \mathcal{A}_{1}:=\left\{f \in L^{2}(a, b): \int_{a}^{b}\left|\mathbb{I}_{b-}^{k, \lambda} f(t)-\lambda \mathbb{I}_{b-}^{k+1, \lambda} f(t)\right|^{2} d t<\infty\right\}$. Here $k=-\sigma$, and $\mathcal{A}_{1}$ is a linear space with inner product $\langle f, g\rangle_{\mathcal{A}_{1}}:=\langle F, G\rangle_{L^{2}(a, b)}$ where

$$
\begin{aligned}
& F(t)=\Gamma(k+1)\left(\mathbb{I}_{b-}^{k, \lambda} f(t)-\lambda \mathbb{I}_{b-}^{k+1, \lambda} f(t)\right), \\
& G(t)=\Gamma(k+1)\left(\mathbb{I}_{b-}^{k, \lambda} g(t)-\lambda \mathbb{I}_{b-}^{k+1, \lambda} g(t)\right) .
\end{aligned}
$$

The following inequalities will be used in the proof of our main results in this section. 
Lemma 1 For any $-1 / 2<\sigma<0$, we have

$$
\begin{gathered}
\int_{0}^{u \wedge r}(u-s)^{-\sigma-1}(r-s)^{-\sigma-1} d s \leq|r-u|^{-2 \sigma-1} \beta(1+2 \sigma,-\sigma) \\
\int_{0}^{x \wedge y}(x-s)^{-\sigma}(y-s)^{-\sigma} d s \leq(x \vee y)^{2}|x-y|^{-2 \sigma-1} \beta(1+2 \sigma, 1-\sigma),
\end{gathered}
$$

where $\beta(\cdot, \cdot)$ is the beta function.

Proof It follows from Lemma 2.2 in [31] that

$$
\int_{0}^{1} t^{u-1}(1-t)^{v-1}(c-t)^{-u-v} d t=c^{-v}(c-1)^{-u} \beta(u, v)
$$

for $u, v>0, c>1$. Consider first the case $u>r$, by (2.7) we obtain

$$
\begin{aligned}
\int_{0}^{u \wedge r}(u-s)^{-\sigma-1}(r-s)^{-\sigma-1} d s & =\int_{0}^{r}(u-s)^{-\sigma-1}(r-s)^{-\sigma-1} d s \\
& \left.=\int_{0}^{1}\left(\frac{u}{r}-y\right)^{-\sigma-1}(1-y)^{-\sigma-1} r^{-2 \sigma-1} d y \quad \text { (change of variable } y=s / r\right) \\
& \leq \int_{0}^{1}\left(\frac{u}{r}-y\right)^{-\sigma-1}(1-y)^{-\sigma-1} y^{2 \sigma} r^{-2 \sigma-1} d y \\
& =\left(\frac{u}{r}-1\right)^{-2 \sigma-1}\left(\frac{u}{r}\right)^{\sigma} \beta(1+2 \sigma,-\sigma) r^{-2 \sigma-1} \\
& =(u-r)^{-2 \sigma-1}\left(\frac{u}{r}\right)^{\sigma} \beta(1+2 \sigma,-\sigma) \leq(u-r)^{-2 \sigma-1} \beta(1+2 \sigma,-\sigma) .
\end{aligned}
$$

For the case $r>u$, in a similar way as above, we have

$$
\int_{0}^{u \wedge r}(u-s)^{-\sigma-1}(r-s)^{-\sigma-1} d s \leq(r-u)^{-2 \sigma-1} \beta(1+2 \sigma,-\sigma),
$$

and consequently

$$
\int_{0}^{u \wedge r}(u-s)^{-\sigma-1}(r-s)^{-\sigma-1} d s \leq|r-u|^{-2 \sigma-1} \beta(1+2 \sigma,-\sigma) .
$$

We want to show now that (2.6) holds true. For the case $x>y$, we deduce from (2.7) that

$$
\begin{aligned}
\int_{0}^{x \wedge y}(x-s)^{-\sigma}(y-s)^{-\sigma} d s & =\int_{0}^{y}(x-s)^{-\sigma}(y-s)^{-\sigma} d s \\
& \left.=y^{-2 \sigma+1} \int_{0}^{1}\left(\frac{x}{y}-t\right)^{-\sigma}(1-t)^{-\sigma} d t \quad \text { (change of variable } t=s / y\right) \\
& =y^{-2 \sigma+1} \int_{0}^{1}\left(\frac{x}{y}-t\right)^{-\sigma-2}(1-t)^{-\sigma}\left(\frac{x}{y}-t\right)^{2} d t
\end{aligned}
$$




$$
\begin{aligned}
& \leq y^{-2 \sigma+1}\left(\frac{x}{y}\right)^{2} \int_{0}^{1}\left(\frac{x}{y}-t\right)^{-\sigma-2}(1-t)^{-\sigma} t^{2 \sigma} d t \\
& =y^{-2 \sigma-1} x^{2}\left(\frac{x}{y}\right)^{\sigma-1}\left(\frac{x}{y}-1\right)^{-2 \sigma-1} \beta(1+2 \sigma, 1-\sigma) \\
& =x^{2}\left(\frac{x}{y}\right)^{\sigma-1}(x-y)^{-2 \sigma-1} \beta(1+2 \sigma, 1-\sigma) \\
& \leq x^{2}(x-y)^{-2 \sigma-1} \beta(1+2 \sigma, 1-\sigma) .
\end{aligned}
$$

For the case $y>x$, using a similar argument as above, we find that

$$
\int_{0}^{x \wedge y}(x-s)^{-\sigma}(y-s)^{-\sigma} d s \leq y^{2}(y-x)^{-2 \sigma-1} \beta(1+2 \sigma, 1-\sigma) .
$$

Thus,

$$
\int_{0}^{x \wedge y}(x-s)^{-\sigma}(y-s)^{-\sigma} d s \leq(x \vee y)^{2}|x-y|^{-2 \sigma-1} \beta(1+2 \sigma, 1-\sigma) .
$$

The proof of this lemma is completed.

Now we state and prove the following important result, which will be needed throughout the paper.

Lemma 2 If $\phi:[0, T] \mapsto \mathcal{L}_{Q}^{0}(\mathcal{U}, \mathcal{H})$ satisfies $\int_{0}^{T}\|\phi(s)\|_{Q}^{2} d s<\infty$, then for any $t \in[0, T]$,

$$
\begin{aligned}
E\left\|\int_{0}^{t} \phi(s) d B_{Q}^{\sigma, \lambda}(s)\right\|^{2} \leq\left((2 H-1) t^{2 H-1} \beta\left(2-2 H, H-\frac{1}{2}\right)\right. \\
\left.+4 \lambda^{2} t^{2 H+1} \frac{\beta\left(2-2 H, H+\frac{1}{2}\right)}{2 H-1}\right) \int_{0}^{t}\|\phi(s)\|_{Q}^{2} d s,
\end{aligned}
$$

where $-1 / 2<\sigma<0, \lambda>0, H=\frac{1}{2}-\sigma$ and $\beta(\cdot, \cdot)$ is the beta function.

Proof Let $\left\{e_{k}\right\}_{k \in \mathbb{N}}$ be the complete orthonormal basis of $\mathcal{U}$ introduced above. By Definition 3 and Lemma 1 we obtain

$$
\begin{aligned}
E & \left\|\int_{0}^{t} \phi(s) d B_{Q}^{\sigma, \lambda}(s)\right\|^{2} \\
& =E\left\|\int_{0}^{t} \sum_{k=1}^{\infty} \phi(s) \sqrt{\lambda_{k}} e_{k} d B_{k}^{\sigma, \lambda}(s)\right\|^{2} \\
& \leq \sum_{k=1}^{\infty} \lambda_{k} E\left|\int_{0}^{t}\left\|\phi(s) e_{k}\right\| d B_{k}^{\sigma, \lambda}(s)\right|^{2} \\
& =\sum_{k=1}^{\infty} \lambda_{k}(\Gamma(1-\sigma))^{2} E\left|\int_{0}^{t}\left(\mathbb{I}_{t-}^{-\sigma, \lambda}\left\|\phi(s) e_{k}\right\|-\lambda \mathbb{I}_{t-}^{1-\sigma, \lambda}\left\|\phi(s) e_{k}\right\|\right) d B_{k}(s)\right|^{2}
\end{aligned}
$$




$$
\begin{aligned}
& =\sum_{k=1}^{\infty} \lambda_{k}(\Gamma(1-\sigma))^{2} E \int_{0}^{t}\left|\mathbb{I}_{t-}^{-\sigma, \lambda}\left\|\phi(s) e_{k}\right\|-\lambda \mathbb{I}_{t-}^{1-\sigma, \lambda}\left\|\phi(s) e_{k}\right\|\right|^{2} d s \\
& \leq \sum_{k=1}^{\infty} 2 \lambda_{k} \int_{0}^{t}\left(\sigma^{2}\left(\int_{s}^{t}\left\|\phi(u) e_{k}\right\|(u-s)^{-\sigma-1} e^{-\lambda(u-s)} d u\right)^{2}\right. \\
& \left.+\lambda^{2}\left(\int_{s}^{t}\left\|\phi(x) e_{k}\right\|(x-s)^{-\sigma} e^{-\lambda(x-s)} d x\right)^{2}\right) d s \\
& =\sum_{k=1}^{\infty} 2 \lambda_{k} \sigma^{2} \int_{0}^{t} \int_{s}^{t} \int_{s}^{t}\left\|\phi(u) e_{k}\right\|\left\|\phi(r) e_{k}\right\|(u-s)^{-\sigma-1}(r-s)^{-\sigma-1} e^{-\lambda(u-s)} e^{-\lambda(r-s)} d u d r d s \\
& +\sum_{k=1}^{\infty} 2 \lambda_{k} \lambda^{2} \int_{0}^{t} \int_{s}^{t} \int_{s}^{t}\left\|\phi(x) e_{k}\right\|\left\|\phi(y) e_{k}\right\|(y-s)^{-\sigma}(x-s)^{-\sigma} e^{-\lambda(y-s)} e^{-\lambda(x-s)} d x d y d s \\
& \leq \sum_{k=1}^{\infty} 2 \lambda_{k} \sigma^{2} \int_{0}^{t} \int_{0}^{t} \int_{0}^{u \wedge r}\left\|\phi(u) e_{k}\right\|\left\|\phi(r) e_{k}\right\|(u-s)^{-\sigma-1}(r-s)^{-\sigma-1} d s d u d r \\
& +\sum_{k=1}^{\infty} 2 \lambda_{k} \lambda^{2} \int_{0}^{t} \int_{0}^{t} \int_{0}^{x \wedge y}\left\|\phi(x) e_{k}\right\|\left\|\phi(y) e_{k}\right\|(y-s)^{-\sigma}(x-s)^{-\sigma} d s d x d y \\
& \leq \sum_{k=1}^{\infty} \lambda_{k}\left(2 \sigma^{2} \int_{0}^{t} \int_{0}^{t}\left\|\phi(r) e_{k}\right\|^{2}|u-r|^{-2 \sigma-1} \beta(1+2 \sigma,-\sigma) d u d r\right. \\
& \left.+2(\lambda t)^{2} \int_{0}^{t} \int_{0}^{t}\left\|\phi(y) e_{k}\right\|^{2}|y-x|^{-2 \sigma-1} \beta(1+2 \sigma, 1-\sigma) d x d y\right) \\
& \leq\left((2 H-1) t^{2 H-1} \beta\left(2-2 H, H-\frac{1}{2}\right)+4 \lambda^{2} t^{2 H+1} \frac{\beta\left(2-2 H, H+\frac{1}{2}\right)}{2 H-1}\right) \int_{0}^{t}\|\phi(s)\|_{Q}^{2} d s .
\end{aligned}
$$

Therefore, we complete the proof of this lemma.

Since $\left\{B_{k}^{H}\right\}_{k \geq 1}$ and $\left\{B_{k}\right\}_{k \geq 1}$, respectively, are the sequences of two-sided one-dimensional standard FBMs and Brownian motions mutually independent on $\left(\Omega, \mathcal{F},\{\mathcal{F}\}_{t \geq 0}, P\right)$, we have the following properties for the stochastic integrals with respect to $B_{Q}^{H}$ and $B_{Q}$ (see, e.g., $[2,4]$ ).

Lemma 3 If $\phi:[0, T] \mapsto \mathcal{L}_{Q}^{0}(\mathcal{U}, \mathcal{H})$ satisfies $\int_{0}^{T}\|\phi(s)\|_{Q}^{2} d s<\infty$, then for any $t \in[0, T]$,

$$
\begin{aligned}
& E\left\|\int_{0}^{t} \phi(s) d B_{Q}^{H}(s)\right\|^{2} \leq 2 H t^{2 H-1} \int_{0}^{t}\|\phi(s)\|_{Q}^{2} d s \\
& E\left\|\int_{0}^{t} \phi(s) d B_{Q}(s)\right\|^{2} \leq \int_{0}^{t}\|\phi(s)\|_{Q}^{2} d s
\end{aligned}
$$

where $H \in(1 / 2,1)$. 


\section{Existence and uniqueness of mild solutions to stochastic evolution equations with unbounded delay and a TFBM}

Let $(\Omega, \mathcal{F}, P)$ be the complete probability space which was introduced in Section 2. Denote $\mathcal{F}_{t}=\mathcal{F}_{0}$, for all $t \leq 0$.

Throughout this paper we shall assume $0 \leq \alpha<1 / 2$ and define the Banach space $D\left(A^{\alpha}\right)$ with the norm $\|y\|_{\alpha}:=\left\|A^{\alpha} y\right\|$ for $y \in D\left(A^{\alpha}\right)$, where $D\left(A^{\alpha}\right)$ denotes the domain of the fractional power operator $A^{\alpha}: \mathcal{H} \rightarrow \mathcal{H}$. Denote $\mathcal{H}^{\alpha}=D\left(A^{\alpha}\right)$. We denote by $C\left(a, b ; L^{2}\left(\Omega ; \mathcal{H}^{\alpha}\right)\right)=C\left(a, b ; L^{2}\left(\Omega, \mathcal{F}, P ; \mathcal{H}^{\alpha}\right)\right)$ the Banach space of all continuous functions from $[a, b]$ into $L^{2}\left(\Omega ; \mathcal{H}^{\alpha}\right)$ equipped with the sup norm.

Let us also consider a real number $T>0$. If $x \in C\left(-\infty, T ; L^{2}\left(\Omega ; \mathcal{H}^{\alpha}\right)\right)$ for each $t \in[0, T]$ we denote by $x_{t} \in C\left(-\infty, 0 ; L^{2}\left(\Omega ; \mathcal{H}^{\alpha}\right)\right)$ the function defined by $x_{t}(s)=x(t+s)$, for $s \in(-\infty, 0]$. We define the abstract phase space $\mathscr{C}\left(\mathcal{H}^{\alpha}\right)$ by

$$
\mathscr{C}\left(\mathcal{H}^{\alpha}\right)=\left\{\psi \in C\left(-\infty, 0 ; L^{2}\left(\Omega ; \mathcal{H}^{\alpha}\right)\right): \lim _{\theta \rightarrow-\infty} \psi(\theta) \text { exists in } L^{2}\left(\Omega ; \mathcal{H}^{\alpha}\right)\right\} .
$$

If $\mathscr{C}\left(\mathcal{H}^{\alpha}\right)$ is endowed with the norm

$$
\|\psi\|_{\mathscr{C}\left(\mathcal{H}^{\alpha}\right)}=\left(\sup _{\theta \in(-\infty, 0]} E\|\psi(\theta)\|_{\alpha}^{2}\right)^{\frac{1}{2}}, \quad \psi \in \mathscr{C}\left(\mathcal{H}^{\alpha}\right)
$$

then $\left(\mathscr{C}\left(\mathcal{H}^{\alpha}\right),\|\cdot\|_{\mathscr{C}\left(\mathcal{H}^{\alpha}\right)}\right)$ is a Banach space.

In this section we consider the global existence and uniqueness of mild solutions to the following stochastic evolution equation with infinite delay:

$$
\left\{\begin{array}{l}
d u(t)=-A u(t) d t+f\left(t, u_{t}\right) d t+g\left(t, u_{t}\right) d B_{Q}^{\sigma, \lambda}(t), \quad t>0 \\
u(t)=\varphi(t), \quad t \in(-\infty, 0]
\end{array}\right.
$$

where $B_{Q}^{\sigma, \lambda}(t)$ is the tempered fractional Brownian motion which was introduced in the previous section, the initial data $\varphi \in \mathscr{C}\left(\mathcal{H}^{\alpha}\right)$ with $\varphi(t)$ being $\mathcal{F}_{t}$-measurable with $\mathcal{F}_{t}=\mathcal{F}_{0}$ for all $t \leq 0,-A$ is the infinitesimal generator of an analytic semigroup $S(t), t \geq 0$, on the separable Hilbert space $\mathcal{H}$. Furthermore, for the closed, densely defined linear operator $-A$ we assume the following conditions:

(A1) There exist a constant $G \geq 1$ and a real number $\delta>0$ such that for any $x \in \mathcal{H}$,

$$
\|S(t) x\| \leq G e^{-\delta t}\|x\|, \quad t \geq 0
$$

(A2) The fractional power $A^{\alpha}$ satisfies that for any $x \in \mathcal{H}$,

$$
\left\|A^{\alpha} S(t) x\right\| \leq G_{\alpha} e^{-\delta t} t^{-\alpha}\|x\|, \quad t>0
$$

where $G_{\alpha} \geq 1$ 
(A3) There exists a constant $Q_{\alpha} \geq 1$ such that for any $x \in \mathcal{H}^{\alpha}$,

$$
\|S(t) x-x\| \leq Q_{\alpha} t^{\alpha}\left\|A^{\alpha} x\right\|, \quad t>0
$$

The delay term $f:[0, \infty) \times \mathscr{C}\left(\mathcal{H}^{\alpha}\right) \mapsto \mathcal{H}$ satisfies

(B1) For any $\xi \in \mathscr{C}\left(\mathcal{H}^{\alpha}\right)$, the mapping $[0, \infty) \ni t \mapsto f(t, \xi) \in \mathcal{H}$ is measurable.

(B2) There exists $l_{1}>0$ such that for any $\xi, \eta \in \mathscr{C}\left(\mathcal{H}^{\alpha}\right)$ and $t \geq 0$,

$$
E\|f(t, \xi)-f(t, \eta)\|^{2} \leq l_{1}\|\xi-\eta\|_{\mathscr{C}\left(\mathcal{H}^{\alpha}\right)}^{2}
$$

(B3) There exists $l_{2}>0$ such that for any $\xi \in \mathscr{C}\left(\mathcal{H}^{\alpha}\right)$ and $t \geq 0$,

$$
E\|f(t, \xi)\|^{2} \leq l_{2}\left(1+\|\xi\|_{\mathscr{C}\left(\mathcal{H}^{\alpha}\right)}^{2}\right)
$$

Moreover, the delay term $g:[0, \infty) \times \mathscr{C}\left(\mathcal{H}^{\alpha}\right) \mapsto \mathcal{L}_{Q}^{0}(\mathcal{U}, \mathcal{H})$ satisfies the following conditions:

$(C 1)$ For any $\xi \in \mathscr{C}\left(\mathcal{H}^{\alpha}\right)$, the mapping $[0, \infty) \ni t \mapsto g(t, \xi) \in \mathcal{L}_{Q}^{0}(\mathcal{U}, \mathcal{H})$ is measurable.

$(C 2)$ There exists a nonnegative function $k_{1} \in L^{\infty}\left(\mathbb{R}^{+}\right)$such that for any $\xi, \eta \in \mathscr{C}\left(\mathcal{H}^{\alpha}\right)$ and $t \geq 0$,

$$
E\|g(t, \xi)-g(t, \eta)\|_{Q}^{2} \leq k_{1}(t)\|\xi-\eta\|_{\mathscr{C}\left(\mathcal{H}^{\alpha}\right)}^{2}
$$

and $\left\|k_{1}\right\|_{L^{\infty}\left(\mathbb{R}^{+}\right)}:=K_{1}<\infty$.

(C3) There exist nonnegative functions $k_{2} \in L^{p}\left(\mathbb{R}^{+}\right)$with $p \in\left(\frac{1}{1-2 \alpha}, \infty\right)$ and $k_{3} \in L^{\infty}\left(\mathbb{R}^{+}\right)$such that for any $\xi \in \mathscr{C}\left(\mathcal{H}^{\alpha}\right)$ and $t \geq 0$

$$
E\|g(t, \xi)\|_{Q}^{2} \leq k_{2}(t)+k_{3}(t)\|\xi\|_{\mathscr{C}\left(\mathcal{H}^{\alpha}\right)}^{2},
$$

and

$$
\int_{0}^{\infty}\left(k_{2}(t)\right)^{p} d t:=K_{2}<\infty, \quad\left\|k_{3}\right\|_{L^{\infty}\left(\mathbb{R}^{+}\right)}:=K_{3}<\infty .
$$

Now we state the definition of mild solution to problem (3.1).

Definition 4 Let $\varphi \in \mathscr{C}\left(\mathcal{H}^{\alpha}\right)$ be an initial process with $\mathcal{F}_{t}=\mathcal{F}_{0}$ for all $t \leq 0$. An $\mathcal{F}_{t}$-adapted stochastic process $u(t)$ is called a mild solution of $(3.1)$ if $u \in C\left(-\infty, T ; L^{2}\left(\Omega ; \mathcal{H}^{\alpha}\right)\right), u(t)=\varphi(t)$ for $t \in(-\infty, 0]$, and for $t \in[0, T]$,

$$
u(t)=S(t) \varphi(0)+\int_{0}^{t} S(t-r) f\left(r, u_{r}\right) d r+\int_{0}^{t} S(t-r) g\left(r, u_{r}\right) d B_{Q}^{\sigma, \lambda}(r) \quad P \text {-a.s. }
$$

Definition 5 Let $\left(\Omega, \mathcal{F},\left\{\mathcal{F}_{t}\right\}_{t \geq 0}, P\right)$ be a filtered probability space. A stochastic process $\{X(t)\}_{t \geq 0}$ is said to be predictable if $X$, considered as a mapping from $\mathbb{R}^{+} \times \Omega$, is measurable with respect to the $\sigma$-algebra generated by all left-continuous $\mathcal{F}_{t}$-adapted processes.

We also need the following lemma. 
Lemma 4 Let $\psi(t): \mathbb{R}^{+} \times \Omega \mapsto \mathcal{L}_{Q}^{0}(\mathcal{U}, \mathcal{H})$ be a predictable, $\mathcal{F}_{t}$-adapted process. If $\psi(t) v \in \mathcal{H}^{\alpha}, t \geq 0$, for any $v \in \mathcal{U}$ and $\int_{0}^{t} E\|\psi(r)\|_{Q}^{2} d r<\infty, \int_{0}^{t} E\left\|A^{\alpha} \psi(r)\right\|_{Q}^{2} d r<\infty$, then

$$
A^{\alpha} \int_{0}^{t} \psi(r) d B_{Q}^{\sigma, \lambda}(r)=\int_{0}^{t} A^{\alpha} \psi(r) d B_{Q}^{\sigma, \lambda}(r) \quad P \text {-a.s. }
$$

Proof By Proposition 4.22 in [11] there exists a sequence $\left\{\psi_{n}\right\}$ of $D\left(A^{\alpha}\right)$-valued predictable processes on $[0, t]$ taking only a finite numbers of values such that

$$
E \int_{0}^{t}\left\|\psi(r)-\psi_{n}(r)\right\|_{Q}^{2} d r+E \int_{0}^{t}\left\|A^{\alpha} \psi(r)-A^{\alpha} \psi_{n}(r)\right\|_{Q}^{2} d r \longrightarrow 0 \text { as } n \rightarrow \infty .
$$

This and Lemma 2 imply that

$$
E\left\|\int_{0}^{t}\left(\psi(r)-\psi_{n}(r)\right) d B_{Q}^{\sigma, \lambda}(r)\right\|^{2}+E\left\|\int_{0}^{t}\left(A^{\alpha} \psi(r)-A^{\alpha} \psi_{n}(r)\right) d B_{Q}^{\sigma, \lambda}(r)\right\|^{2} \longrightarrow 0,
$$

as $n \rightarrow \infty$. From the definition of the integral, we have

$$
A^{\alpha} \int_{0}^{t} \psi_{n}(r) d B_{Q}^{\sigma, \lambda}(r)=\int_{0}^{t} A^{\alpha} \psi_{n}(r) d B_{Q}^{\sigma, \lambda}(r)
$$

Thanks to (3.4)-(3.5) and the closedness of $A^{\alpha}$, we deduce that

$$
A^{\alpha} \int_{0}^{t} \psi(r) d B_{Q}^{\sigma, \lambda}(r)=\int_{0}^{t} A^{\alpha} \psi(r) d B_{Q}^{\sigma, \lambda}(r) \quad P \text {-a.s. }
$$

We now introduce the following notation. Let $u \in C\left(0, T ; L^{2}\left(\Omega ; \mathcal{H}^{\alpha}\right)\right)$ with $u(0)=\varphi(0)$ and $\varphi \in \mathscr{C}\left(\mathcal{H}^{\alpha}\right)$. Then for $r \in[0, T]$, we denote by $u \vee_{r} \varphi$ the mapping from $\mathbb{R}^{-}$to $L^{2}\left(\Omega ; \mathcal{H}^{\alpha}\right)$ defined by

$$
u \vee_{r} \varphi(s)= \begin{cases}u(r+s), & s \in(-r, 0] \\ \varphi(r+s), & s \leq-r\end{cases}
$$

It follows from [5] that, for such function $u$, the integral in (3.2) is well defined.

Theorem 1 Let $0<\alpha<\frac{1}{2}$. Suppose that assumptions $(A 1)-(A 3),(B 1)-(B 3)$ and $(C 1)-(C 3)$ hold. Then for each $\varphi \in \mathscr{C}\left(\mathcal{H}^{\alpha}\right)$ there exists a unique local mild solution $u$ to $(3.1)$ on $[0, h]$ for some $h>0$.

Proof Let us fix some $\varphi \in \mathscr{C}\left(\mathcal{H}^{\alpha}\right)$, and let $R:=3 G^{2}\left(E\|\varphi(0)\|_{\alpha}^{2}+1\right)$. Assume $h \in(0, T)$ is a fixed time which has been chosen such that

$$
\begin{array}{r}
3 G_{\alpha}^{2} l_{2}\left(1+R+\|\varphi\|_{\mathscr{C}\left(\mathcal{H}^{\alpha}\right)}^{2}\right) \frac{h^{2-2 \alpha}}{1-2 \alpha}+3\left((2 H-1) h^{2 H-1} \beta\left(2-2 H, H-\frac{1}{2}\right)+4 \lambda^{2} h^{2 H+1}\right. \\
\left.\frac{\beta\left(2-2 H, H+\frac{1}{2}\right)}{2 H-1}\right) G_{\alpha}^{2}\left(K_{3}\left(R+\|\varphi\|_{\mathscr{C}\left(\mathcal{H}^{\alpha}\right)}^{2}\right) \frac{h^{1-2 \alpha}}{1-2 \alpha}+\left(\frac{h^{1-2 \alpha q}}{1-2 \alpha q}\right)^{\frac{1}{q}} K_{2}^{\frac{1}{p}}\right) \leq 3 G^{2},
\end{array}
$$


and

$$
\begin{aligned}
2 G_{\alpha}^{2} l_{1} & \frac{h^{1-2 \alpha}}{1-2 \alpha}+2\left((2 H-1) h^{2 H-1} \beta\left(2-2 H, H-\frac{1}{2}\right)+4 \lambda^{2} h^{2 H+1}\right. \\
& \left.\times \frac{\beta\left(2-2 H, H+\frac{1}{2}\right)}{2 H-1}\right) G_{\alpha}^{2} K_{1} \frac{h^{1-2 \alpha}}{1-2 \alpha}<1 .
\end{aligned}
$$

Consider

$$
B(R)=\left\{u \in C\left(0, h ; L^{2}\left(\Omega ; \mathcal{H}^{\alpha}\right)\right): u(0)=\varphi(0), \sup _{t \in[0, h]} E\|u(t)\|_{\alpha}^{2} \leq R\right\} .
$$

$B(R)$ is a bounded set in $C\left(0, h ; L^{2}\left(\Omega ; \mathcal{H}^{\alpha}\right)\right)$. We introduce the mapping $\Phi$ defined by

$$
(\Phi u)(t)=S(t) \varphi(0)+\int_{0}^{t} S(t-r) f\left(r, u \vee_{r} \varphi\right) d r+\int_{0}^{t} S(t-r) g\left(r, u \vee_{r} \varphi\right) d B_{Q}^{\sigma, \lambda}(r), \quad t \in[0, h]
$$

We split the proof into three steps.

Step 1. $\Phi$ maps $B(R)$ into $C\left(0, h ; L^{2}\left(\Omega ; \mathcal{H}^{\alpha}\right)\right)$.

Let $0<t<h$ and $u \in B(R)$ be given arbitrarily. Then for $\tau>0$ small enough, we have

$$
\begin{aligned}
& E\|(\Phi u)(t+\tau)-(\Phi u)(t)\|_{\alpha}^{2} \\
& \leq 5 E\|S(t+\tau) \varphi(0)-S(t) \varphi(0)\|_{\alpha}^{2} \\
& \quad+5 E\left\|\int_{0}^{t}(S(t+\tau-r)-S(t-r)) f\left(r, u \vee_{r} \varphi\right) d r\right\|_{\alpha}^{2} \\
& \quad+5 E\left\|\int_{t}^{t+\tau} S(t+\tau-r) f\left(r, u \vee_{r} \varphi\right) d r\right\|_{\alpha}^{2} \\
& \quad+5 E\left\|\int_{t}^{t+\tau} S(t+\tau-r) g\left(r, u \vee_{r} \varphi\right) d B_{Q}^{\sigma, \lambda}(r)\right\|_{\alpha}^{2} \\
& \quad+5 E\left\|\int_{0}^{t}(S(t+\tau-r)-S(t-r)) g\left(r, u \vee_{r} \varphi\right) d B_{Q}^{\sigma, \lambda}(r)\right\|_{\alpha}^{2} \\
& \quad:=I_{1}+I_{2}+I_{3}+I_{4}+I_{5} .
\end{aligned}
$$

Using conditions $(A 2)-(A 3)$, we obtain

$$
\begin{aligned}
I_{1} & =5 E\left\|A^{\alpha}(S(t) S(\tau) \varphi(0)-S(t) \varphi(0))\right\|^{2} \\
& \leq 5 G_{\alpha}^{2} e^{-2 \delta t} t^{-2 \alpha} E\|S(\tau) \varphi(0)-\varphi(0)\|^{2} \\
& \leq 5 G_{\alpha}^{2} Q_{\alpha}^{2} e^{-2 \delta t} t^{-2 \alpha} \tau^{2 \alpha}\|\varphi\|_{\mathscr{C}\left(\mathcal{H}^{\alpha}\right)}^{2} \longrightarrow 0 \quad \text { as } \tau \rightarrow 0 .
\end{aligned}
$$


Let $\varepsilon>0$ be given arbitrarily. Then by Lemma 2, conditions $(A 1)-(A 3),(B 3),(C 3)$ and Hölder's inequality, we can choose $\tau$ and $\eta$ sufficiently small such that

$$
\begin{aligned}
& I_{2} \leq 10 E\left\|\int_{t-\eta}^{t} A^{\alpha} S(t-r)(S(\tau)-I) f\left(r, u \vee_{r} \varphi\right) d r\right\|^{2} \\
& +10 E\left\|\int_{0}^{t-\eta} A^{\alpha} S(t-r-\eta)(S(\tau)-I) S(\eta) f\left(r, u \vee_{r} \varphi\right) d r\right\|^{2} \\
& \leq 10 G_{\alpha}^{2} E\left(\int_{t-\eta}^{t} e^{-\delta(t-r)}(t-r)^{-\alpha}\left\|(S(\tau)-I) f\left(r, u \vee_{r} \varphi\right)\right\| d r\right)^{2} \\
& +10 G_{\alpha}^{2} Q_{\alpha}^{2} E\left(\int_{0}^{t-\eta} e^{-\delta(t-r-\eta)}(t-r-\eta)^{-\alpha} \tau^{\alpha}\left\|A^{\alpha} S(\eta) f\left(r, u \vee_{r} \varphi\right)\right\| d r\right)^{2} \\
& \leq 10 G_{\alpha}^{2} E \int_{t-\eta}^{t}\left\|(S(\tau)-I) f\left(r, u \vee_{r} \varphi\right)\right\|^{2} d r \int_{t-\eta}^{t}(t-r)^{-2 \alpha} d r \\
& +10 G_{\alpha}^{2} Q_{\alpha}^{2} \tau^{2 \alpha} E \int_{0}^{t-\eta}\left\|A^{\alpha} S(\eta) f\left(r, u \vee_{r} \varphi\right)\right\|^{2} d r \int_{0}^{t-\eta}(t-r-\eta)^{-2 \alpha} d r \\
& \leq 10 G_{\alpha}^{2}(G+1)^{2} l_{2}\left(1+R+\|\varphi\|_{\mathscr{C}\left(\mathcal{H}^{\alpha}\right)}^{2}\right) \frac{\eta^{2-2 \alpha}}{1-2 \alpha} \\
& +10 G_{\alpha}^{4} Q_{\alpha}^{2} l_{2}\left(1+R+\|\varphi\|_{\mathscr{C}\left(\mathcal{H}^{\alpha}\right)}^{2}\right) \tau^{2 \alpha} \eta^{-2 \alpha} \frac{(t-\eta)^{2-2 \alpha}}{1-2 \alpha}<\varepsilon, \\
& I_{5} \leq 5 N_{t} \int_{t-\eta}^{t} E\left\|A^{\alpha} S(t-r)(S(\tau)-I) g\left(r, u \vee_{r} \varphi\right)\right\|_{Q}^{2} d r \\
& +5 N_{t} \int_{0}^{t-\eta} E\left\|A^{\alpha} S(t-r-\eta)(S(\tau)-I) S(\eta) g\left(r, u \vee_{r} \varphi\right)\right\|_{Q}^{2} d r \\
& \leq 5 N_{t} G_{\alpha}^{2} \int_{t-\eta}^{t} e^{-2 \delta(t-r)}(t-r)^{-2 \alpha} E\left\|(S(\tau)-I) g\left(r, u \vee_{r} \varphi\right)\right\|_{Q}^{2} d r \\
& +5 N_{t} G_{\alpha}^{2} Q_{\alpha}^{2} \int_{0}^{t-\eta} e^{-2 \delta(t-r-\eta)}(t-r-\eta)^{-2 \alpha} \tau^{2 \alpha} E\left\|A^{\alpha} S(\eta) g\left(r, u \vee_{r} \varphi\right)\right\|_{Q}^{2} d r \\
& \leq 5 N_{t} G_{\alpha}^{2}(G+1)^{2} \int_{t-\eta}^{t}(t-r)^{-2 \alpha}\left(k_{2}(r)+k_{3}(r)\left\|u \vee_{r} \varphi\right\|_{\mathscr{C}\left(\mathcal{H}^{\alpha}\right)}^{2}\right) d r \\
& +5 N_{t} G_{\alpha}^{4} Q_{\alpha}^{2} \int_{0}^{t-\eta}(t-r-\eta)^{-2 \alpha} \tau^{2 \alpha} \eta^{-2 \alpha}\left(k_{2}(r)+k_{3}(r)\left\|u \vee_{r} \varphi\right\|_{\mathscr{C}\left(\mathcal{H}^{\alpha}\right)}^{2}\right) d r \\
& \leq 5 N_{t} G_{\alpha}^{2}(G+1)^{2}\left(\frac{\eta^{1-2 \alpha q}}{1-2 \alpha q}\right)^{\frac{1}{q}} K_{2}^{\frac{1}{p}}+5 N_{t} G_{\alpha}^{2}(G+1)^{2} K_{3}\left(R+\|\varphi\|_{\mathscr{C}\left(\mathcal{H}^{\alpha}\right)}^{2}\right) \frac{\eta^{1-2 \alpha}}{1-2 \alpha} \\
& +5 N_{t} G_{\alpha}^{4} Q_{\alpha}^{2} \tau^{2 \alpha} \eta^{-2 \alpha}\left(\frac{(t-\eta)^{1-2 \alpha q}}{1-2 \alpha q}\right)^{\frac{1}{q}} K_{2}^{\frac{1}{p}} \\
& +5 N_{t} G_{\alpha}^{4} Q_{\alpha}^{2} \tau^{2 \alpha} \eta^{-2 \alpha}\left(R+\|\varphi\|_{\mathscr{C}\left(\mathcal{H}^{\alpha}\right)}^{2}\right) \frac{(t-\eta)^{1-2 \alpha}}{1-2 \alpha}<\varepsilon,
\end{aligned}
$$


where $\frac{1}{q}+\frac{1}{p}=1, p$ is given in condition $(C 3)$, and we have used the notation

$$
N_{t}:=(2 H-1) t^{2 H-1} \beta\left(2-2 H, H-\frac{1}{2}\right)+4 \lambda^{2} t^{2 H+1} \frac{\beta\left(2-2 H, H+\frac{1}{2}\right)}{2 H-1} .
$$

For $I_{3}$ and $I_{4}$, in a similar way as above, we find that

$$
\begin{aligned}
I_{3} & \leq 5 G_{\alpha}^{2} E\left(\int_{t}^{t+\tau} e^{-\delta(t+\tau-r)}(t+\tau-r)^{-\alpha}\left\|f\left(r, u \vee_{r} \varphi\right)\right\| d r\right)^{2} \\
& \leq 5 G_{\alpha}^{2} E \int_{t}^{t+\tau}\left\|f\left(r, u \vee_{r} \varphi\right)\right\|^{2} d r \int_{t}^{t+\tau}(t+\tau-r)^{-2 \alpha} d r \\
& \leq 5 G_{\alpha}^{2} l_{2}\left(1+R+\|\varphi\|_{\mathscr{C}\left(\mathcal{H}^{\alpha}\right)}^{2}\right) \frac{\tau^{2-2 \alpha}}{1-2 \alpha} \longrightarrow 0 \quad \text { as } \tau \rightarrow 0,
\end{aligned}
$$

and

$$
\begin{aligned}
I_{4} \leq & 5 N_{\tau} \int_{t}^{t+\tau} E\left\|A^{\alpha} S(t+\tau-r) g\left(r, u \vee_{r} \varphi\right)\right\|_{Q}^{2} d r \\
\leq & 5 N_{\tau} G_{\alpha}^{2} \int_{t}^{t+\tau} e^{-2 \delta(t+\tau-r)}(t+\tau-r)^{-2 \alpha} E\left\|g\left(r, u \vee_{r} \varphi\right)\right\|_{Q}^{2} d r \\
\leq & 5 N_{\tau} G_{\alpha}^{2} \int_{t}^{t+\tau}(t+\tau-r)^{-2 \alpha}\left(k_{2}(r)+k_{3}(r)\left\|u \vee_{r} \varphi\right\|_{\mathscr{C}\left(\mathcal{H}^{\alpha}\right)}^{2}\right) d r \\
\leq & 5 N_{\tau} G_{\alpha}^{2}\left(\frac{\tau^{1-2 \alpha q}}{1-2 \alpha q}\right)^{\frac{1}{q}} K_{2}^{\frac{1}{p}} \\
& +5 N_{\tau} G_{\alpha}^{2} K_{3}\left(R+\|\varphi\|_{\mathscr{C}\left(\mathcal{H}^{\alpha}\right)}^{2}\right) \frac{\tau^{1-2 \alpha}}{1-2 \alpha} \longrightarrow 0 \text { as } \tau \rightarrow 0,
\end{aligned}
$$

where $q$ and $N_{\tau}$ are given in (3.10). Thus, it follows from (3.7)-(3.12) that $E\|(\Phi u)(t+\tau)-(\Phi u)(t)\|_{\alpha}^{2}$ tends to zero as $\tau \rightarrow 0$, and consequently $\Phi u \in C\left(0, h ; L^{2}\left(\Omega ; \mathcal{H}^{\alpha}\right)\right)$.

Step 2. $\Phi$ maps $B(R)$ into itself.

Let $u \in B(R)$. Then we have for $t \in[0, h]$,

$$
\begin{aligned}
& E\|(\Phi u)(t)\|_{\alpha}^{2} \leq 3 E\|S(t) \varphi(0)\|_{\alpha}^{2}+3 E\left\|\int_{0}^{t} S(t-r) f\left(r, u \vee_{r} \varphi\right) d r\right\|_{\alpha}^{2} \\
& \quad+3 E\left\|\int_{0}^{t} S(t-r) g\left(r, u \vee_{r} \varphi\right) d B_{Q}^{\sigma, \lambda}(r)\right\|_{\alpha}^{2}:=I_{6}+I_{7}+I_{8} .
\end{aligned}
$$

Thanks to conditions $(A 1)-(A 2)$ and $(B 3)$, we obtain

$$
I_{6} \leq 3 G^{2} e^{-2 \delta t} E\|\varphi(0)\|_{\alpha}^{2} \leq 3 G^{2} E\|\varphi(0)\|_{\alpha}^{2},
$$


and

$$
\begin{aligned}
I_{7} & \leq 3 G_{\alpha}^{2} E\left(\int_{0}^{t} e^{-\delta(t-r)}(t-r)^{-\alpha}\left\|f\left(r, u \vee_{r} \varphi\right)\right\| d r\right)^{2} \\
& \leq 3 G_{\alpha}^{2} E \int_{0}^{t}\left\|f\left(r, u \vee_{r} \varphi\right)\right\|^{2} d r \int_{0}^{t}(t-r)^{-2 \alpha} d r \\
& \leq 3 G_{\alpha}^{2} l_{2}\left(1+R+\|\varphi\|_{\mathscr{C}\left(\mathcal{H}^{\alpha}\right)}^{2}\right) \frac{t^{2-2 \alpha}}{1-2 \alpha}
\end{aligned}
$$

Applying Lemma 2 to $I_{8}$, we deduce from conditions $(A 2),(C 3)$ and Hölder's inequality that

$$
\begin{aligned}
I_{8} & \leq 3 N_{t} \int_{0}^{t} E\left\|A^{\alpha} S(t-r) g\left(r, u \vee_{r} \varphi\right)\right\|_{Q}^{2} d r \\
& \leq 3 N_{t} G_{\alpha}^{2} \int_{0}^{t} e^{-2 \delta(t-r)}(t-r)^{-2 \alpha}\left(k_{2}(r)+k_{3}(r)\left\|u \vee_{r} \varphi\right\|_{\mathscr{C}\left(\mathcal{H}^{\alpha}\right)}^{2}\right) d r \\
& \leq 3 N_{t} G_{\alpha}^{2}\left(\frac{t^{1-2 \alpha q}}{1-2 \alpha q}\right)^{\frac{1}{q}} K_{2}^{\frac{1}{p}}+3 N_{t} G_{\alpha}^{2} K_{3}\left(R+\|\varphi\|_{\mathscr{C}\left(\mathcal{H}^{\alpha}\right)}^{2}\right) \frac{t^{1-2 \alpha}}{1-2 \alpha}
\end{aligned}
$$

where $q$ and $N_{t}$ are given in (3.10). Hence,

$$
\begin{aligned}
\sup _{t \in[0, h]} E & \|(\Phi u)(t)\|_{\alpha}^{2} \leq 3 G^{2} E\|\varphi(0)\|_{\alpha}^{2}+3 G_{\alpha}^{2} l_{2}\left(1+R+\|\varphi\|_{\mathscr{C}\left(\mathcal{H}^{\alpha}\right)}^{2}\right) \frac{h^{2-2 \alpha}}{1-2 \alpha} \\
+ & 3\left((2 H-1) h^{2 H-1} \beta\left(2-2 H, H-\frac{1}{2}\right)+4 \lambda^{2} h^{2 H+1} \frac{\beta\left(2-2 H, H+\frac{1}{2}\right)}{2 H-1}\right) \\
& \times G_{\alpha}^{2}\left(K_{3}\left(R+\|\varphi\|_{\mathscr{C}\left(\mathcal{H}^{\alpha}\right)}^{2}\right) \frac{h^{1-2 \alpha}}{1-2 \alpha}+\left(\frac{h^{1-2 \alpha q}}{1-2 \alpha q}\right)^{\frac{1}{q}} K_{2}^{\frac{1}{p}}\right) \leq R .
\end{aligned}
$$

Step 3. We show that $\Phi: B(R) \mapsto B(R)$ is a contraction mapping. 
Let $u, v \in B(R)$, then we obtain that for any $t \in[0, h]$,

$$
\begin{aligned}
E \|(\Phi u)(t)- & (\Phi v)(t)\left\|_{\alpha}^{2} \leq 2 E\right\| \int_{0}^{t} S(t-r)\left(f\left(r, u \vee_{r} \varphi\right)-f\left(r, v \vee_{r} \varphi\right)\right) d r \|_{\alpha}^{2} \\
& +2 E\left\|\int_{0}^{t} S(t-r)\left(g\left(r, u \vee_{r} \varphi\right)-g\left(r, v \vee_{r} \varphi\right)\right) d B_{Q}^{\sigma, \lambda}(r)\right\|_{\alpha}^{2} \\
\leq & 2 G_{\alpha}^{2} E\left(\int_{0}^{t} e^{-\delta(t-r)}(t-r)^{-\alpha}\left\|f\left(r, u \vee_{r} \varphi\right)-f\left(r, v \vee_{r} \varphi\right)\right\| d r\right)^{2} \\
& +2 N_{t} \int_{0}^{t} E\left\|A^{\alpha} S(t-r)\left(g\left(r, u \vee_{r} \varphi\right)-g\left(r, v \vee_{r} \varphi\right)\right)\right\|_{Q}^{2} d r \\
\leq & 2 G_{\alpha}^{2} E \int_{0}^{t}\left\|f\left(r, u \vee_{r} \varphi\right)-f\left(r, v \vee_{r} \varphi\right)\right\|^{2} d r \int_{0}^{t}(t-r)^{-2 \alpha} d r \\
& +2 N_{t} G_{\alpha}^{2} \int_{0}^{t} e^{-2 \delta(t-r)}(t-r)^{-2 \alpha} E\left\|g\left(r, u \vee_{r} \varphi\right)-g\left(r, v \vee_{r} \varphi\right)\right\|_{Q}^{2} d r \\
\leq & 2 G_{\alpha}^{2} l_{1} \frac{t^{1-2 \alpha}}{1-2 \alpha} \sup _{r \in[0, t]} E\|u(r)-v(r)\|_{\alpha}^{2} \\
& +2 N_{t} G_{\alpha}^{2} K_{1} \frac{t^{1-2 \alpha}}{1-2 \alpha} \sup _{r \in[0, t]} E\|u(r)-v(r)\|_{\alpha}^{2},
\end{aligned}
$$

due to conditions $(A 2),(B 2),(C 2)$ and Hölder's inequality, where $N_{t}$ and $q$ are given in (3.10). This implies that

$$
\sup _{t \in[0, h]} E\|(\Phi u)(t)-(\Phi v)(t)\|_{\alpha}^{2} \leq\left(2 G_{\alpha}^{2} l_{1} \frac{h^{1-2 \alpha}}{1-2 \alpha}+2 N_{h} G_{\alpha}^{2} K_{1} \frac{h^{1-2 \alpha}}{1-2 \alpha}\right) \sup _{t \in[0, h]} E\|u(t)-v(t)\|_{\alpha}^{2} .
$$

Therefore, by the Banach fixed point theorem we obtain the existence of a unique local mild solution to (3.1) on $[0, h]$, and thus the proof of this theorem is completed.

Now we show the global existence of mild solutions to (3.1).

Theorem 2 Let $0<\alpha<\frac{1}{2}$ and assume that assumptions $(A 1)-(A 3),(B 1)-(B 3)$ and $(C 1)-(C 3)$ hold. Then for each $\varphi \in \mathscr{C}\left(\mathcal{H}^{\alpha}\right)$ there exists a unique global mild solution $u(t)$ to (3.1).

Proof For any initial data $\varphi \in \mathscr{C}\left(\mathcal{H}^{\alpha}\right)$, it follows from Theorem 1 that there exists a unique local mild solution $u$ to (3.1). Consider

$$
\mathbb{H}(\omega):=\{T \in[0, \infty): u(\cdot, \omega) \text { is a unique local mild solution to }(3.1) \text { on }[0, T]\}
$$

Let $\sup \mathbb{H}(\omega)=T_{\max }(\omega)$. To show that $u(\cdot)$ is a global mild solution, we need to prove that $T_{\max }=\infty$ a.s.

For sufficiently large $k$, let us define the stopping time

$$
t_{k}(\omega)=\inf \left\{t \in\left[0, T_{\max }(\omega)\right):\|u(t, \omega)\|_{\alpha}>k\right\}
$$


with the usual convention $\inf \emptyset:=\infty$, where $\emptyset$ denotes the empty set. It is clear that $t_{k}$ is a nondecreasing sequence and $t_{k} \rightarrow t_{\infty} \leq T_{\max }$ almost surely as $k \rightarrow \infty$. If we can show that $t_{\infty}=\infty$ a.s., then $T_{\max }=\infty$ a.s., which implies that $u(t)$ is globally defined. Since the sequence $t_{k}$ is increasing, $t_{\infty}=\infty$ a.s. is equivalent to proving that for any $\widetilde{T}>0, P\left(t_{k} \leq \widetilde{T}\right) \rightarrow 0$ as $k \rightarrow \infty$.

By conditions $(A 1)-(A 2),(B 3),(C 1)-(C 3)$, Hölder's inequality and Lemma 2 , we find that for any $t \in$ $[0, \widetilde{T}]$

$$
\begin{aligned}
& E\left\|u\left(t \wedge t_{k}\right)\right\|_{\alpha}^{2} \\
& \leq 3 E\left\|S\left(t \wedge t_{k}\right) \varphi(0)\right\|_{\alpha}^{2}+3 E\left\|\int_{0}^{t \wedge t_{k}} S\left(t \wedge t_{k}-r\right) f\left(r, u \vee_{r} \varphi\right) d r\right\|_{\alpha}^{2} \\
& +3 E\left\|\int_{0}^{t \wedge t_{k}} S\left(t \wedge t_{k}-r\right) g\left(r, u \vee_{r} \varphi\right) d B_{Q}^{\sigma, \lambda}(r)\right\|_{\alpha}^{2} \\
& \leq 3 G^{2} E\|\varphi(0)\|_{\alpha}^{2}+3 G_{\alpha}^{2} E\left(\int_{0}^{t \wedge t_{k}} e^{-\delta\left(t \wedge t_{k}-r\right)}\left(t \wedge t_{k}-r\right)^{-\alpha}\left\|f\left(r, u \vee_{r} \varphi\right)\right\| d r\right)^{2} \\
& +3 N_{t \wedge t_{k}} G_{\alpha}^{2} \int_{0}^{t \wedge t_{k}} e^{-2 \delta\left(t \wedge t_{k}-r\right)}\left(t \wedge t_{k}-r\right)^{-2 \alpha} E\left\|g\left(r, u \vee_{r} \varphi\right)\right\|_{Q}^{2} d r \\
& \leq 3 G^{2} E\|\varphi(0)\|_{\alpha}^{2}+3 G_{\alpha}^{2} \frac{\left(t \wedge t_{k}\right)^{1-2 \alpha}}{1-2 \alpha} l_{2} \int_{0}^{t \wedge t_{k}}\left(1+\|\varphi\|_{\mathscr{C}\left(\mathcal{H}^{\alpha}\right)}^{2}+\sup _{s \in[0, r]} E\|u(s)\|_{\alpha}^{2}\right) d r \\
& +3 N_{t \wedge t_{k}} G_{\alpha}^{2} \int_{0}^{t \wedge t_{k}}\left(t \wedge t_{k}-r\right)^{-2 \alpha}\left(k_{2}(r)+k_{3}(r)\|\varphi\|_{\mathscr{C}\left(\mathcal{H}^{\alpha}\right)}^{2}+k_{3}(r) \sup _{s \in[0, r]} E\|u(s)\|_{\alpha}^{2}\right) d r \\
& \leq 3 G^{2} E\|\varphi(0)\|_{\alpha}^{2}+3 G_{\alpha}^{2} l_{2}\left(1+\|\varphi\|_{\mathscr{C}\left(\mathcal{H}^{\alpha}\right)}^{2}\right) \frac{\widetilde{T}^{2-2 \alpha}}{1-2 \alpha} \\
& +3 G_{\alpha}^{2} l_{2} \frac{\widetilde{T}^{1-2 \alpha}}{1-2 \alpha} \int_{0}^{t} \sup _{s \in[0, r]} E\left\|u\left(s \wedge t_{k}\right)\right\|_{\alpha}^{2} d r+3 N_{\widetilde{T}} G_{\alpha}^{2}\left(\frac{\widetilde{T}^{1-2 \alpha q}}{1-2 \alpha q}\right)^{\frac{1}{q}} K_{2}^{\frac{1}{p}} \\
& +3 N_{\widetilde{T}} G_{\alpha}^{2} K_{3}\|\varphi\|_{\mathscr{C}\left(\mathcal{H}^{\alpha}\right)}^{2} \frac{\widetilde{T}^{1-2 \alpha}}{1-2 \alpha}+3 N_{\widetilde{T}} G_{\alpha}^{2} K_{3} \int_{0}^{t \wedge t_{k}}\left(t \wedge t_{k}-r\right)^{-2 \alpha} \sup _{s \in[0, r]} E\|u(s)\|_{\alpha}^{2} d r
\end{aligned}
$$

which implies that

$$
\begin{aligned}
E\left\|u\left(t \wedge t_{k}\right)\right\|_{\alpha}^{2} \leq & \Pi_{1 \widetilde{T}}+3 G_{\alpha}^{2} l_{2} \frac{\widetilde{T}^{1-2 \alpha+\frac{1}{q}}}{1-2 \alpha}\left(\int_{0}^{t}\left(\sup _{s \in[0, r]} E\left\|u\left(s \wedge t_{k}\right)\right\|_{\alpha}^{2}\right)^{p} d r\right)^{\frac{1}{p}} \\
& +3 N_{\widetilde{T}} G_{\alpha}^{2} K_{3}\left(\frac{\widetilde{T}^{1-2 \alpha q}}{1-2 \alpha q}\right)^{\frac{1}{q}}\left(\int_{0}^{t \wedge t_{k}}\left(\sup _{s \in[0, r]} E\|u(s)\|_{\alpha}^{2}\right)^{p} d r\right)^{\frac{1}{p}}
\end{aligned}
$$


where we have used the notation

$$
\begin{aligned}
\Pi_{1 \widetilde{T}}:=3 G^{2} E & \|\varphi(0)\|_{\alpha}^{2}+3 G_{\alpha}^{2} l_{2}\left(1+\|\varphi\|_{\mathscr{C}\left(\mathcal{H}^{\alpha}\right)}^{2}\right) \frac{\widetilde{T}^{2-2 \alpha}}{1-2 \alpha} \\
& +3 N_{\widetilde{T}} G_{\alpha}^{2}\left(\frac{\widetilde{T}^{1-2 \alpha q}}{1-2 \alpha q}\right)^{\frac{1}{q}} K_{2}^{\frac{1}{p}}+3 N_{\widetilde{T}} G_{\alpha}^{2} K_{3}\|\varphi\|_{\mathscr{C}\left(\mathcal{H}^{\alpha}\right)}^{2} \frac{\widetilde{T}^{1-2 \alpha}}{1-2 \alpha} .
\end{aligned}
$$

It follows from (3.19) that for any $t \in[0, \widetilde{T}]$,

$$
\begin{aligned}
\left(\sup _{s \in[0, t]} E\left\|u\left(s \wedge t_{k}\right)\right\|_{\alpha}^{2}\right)^{p} \leq & 3^{p-1}\left(\Pi_{1 \widetilde{T}}\right)^{p} \\
& +3^{p} G_{\alpha}^{2 p} l_{2}^{p}\left(\frac{\widetilde{T}^{1-2 \alpha+\frac{1}{q}}}{1-2 \alpha}\right)^{p} \int_{0}^{t}\left(\sup _{s \in[0, r]} E\left\|u\left(s \wedge t_{k}\right)\right\|_{\alpha}^{2}\right)^{p} d r \\
& +3^{p} N_{\widetilde{T}}^{p} G_{\alpha}^{2 p} K_{3}^{p}\left(\frac{\widetilde{T}^{1-2 \alpha q}}{1-2 \alpha q}\right)^{\frac{p}{q}} \int_{0}^{t \wedge t_{k}}\left(\sup _{s \in[0, r]} E\|u(s)\|_{\alpha}^{2}\right)^{p} d r \\
= & 3^{p-1}\left(\Pi_{1 \widetilde{T}}\right)^{p}+\Pi_{2 \widetilde{T}} \int_{0}^{t}\left(\sup _{s \in[0, r]} E\left\|u\left(s \wedge t_{k}\right)\right\|_{\alpha}^{2}\right)^{p} d r,
\end{aligned}
$$

where

$$
\Pi_{2 \widetilde{T}}:=3^{p} G_{\alpha}^{2 p} l_{2}^{p}\left(\frac{\widetilde{T}^{1-2 \alpha+\frac{1}{q}}}{1-2 \alpha}\right)^{p}+3^{p} N_{\widetilde{T}}^{p} G_{\alpha}^{2 p} K_{3}^{p}\left(\frac{\widetilde{T}^{1-2 \alpha q}}{1-2 \alpha q}\right)^{\frac{p}{q}} .
$$

Applying Gronwall's lemma to (3.20) we obtain that for all $t \in[0, \widetilde{T}]$,

$$
\left(\sup _{s \in[0, t]} E\left\|u\left(s \wedge t_{k}\right)\right\|_{\alpha}^{2}\right)^{p} \leq 3^{p-1} \Pi_{1 \widetilde{T}}^{p} e^{\Pi_{2} \widetilde{T}^{t}}
$$

and consequently,

$$
\sup _{s \in[0, \widetilde{T}]} E\left\|u\left(s \wedge t_{k}\right)\right\|_{\alpha}^{2} \leq 3^{\frac{p-1}{p}} \Pi_{1 \widetilde{T}} e^{\frac{\Pi_{2} \widetilde{T}}{p}} .
$$

According to the definition of $t_{k},\left\|u\left(t_{k}\right)\right\|_{\alpha}=k$. This implies

$$
\begin{aligned}
k^{2} P\left(t_{k} \leq \widetilde{T}\right) & \leq E\left\|u\left(t_{k}\right)\right\|_{\alpha}^{2} I_{\left\{t_{k} \leq \widetilde{T}\right\}}=E\left\|u\left(\widetilde{T} \wedge t_{k}\right)\right\|_{\alpha}^{2} I_{\left\{t_{k} \leq \widetilde{T}\right\}} \\
& \leq E\left\|u\left(\widetilde{T} \wedge t_{k}\right)\right\|_{\alpha}^{2} \leq 3^{\frac{p-1}{p}} \Pi_{1 \widetilde{T}} e^{\frac{\Pi_{2 \widetilde{T} \widetilde{T}}}{p}}
\end{aligned}
$$

Since $\Pi_{1 \widetilde{T}}$ and $\Pi_{2 \widetilde{T}}$ are independent of $k$, we have $\lim _{k \rightarrow \infty} P\left(t_{k} \leq \widetilde{T}\right)=0$. This implies that (3.1) has a unique global solution $u(t)$ on $[0, \infty)$.

Thanks to Lemma 3, the following result is obtained by similar arguments to those in theorems 1 and 2 . 
Corollary 1 Let $0<\alpha<\frac{1}{2}$ and assume that assumptions $(A 1)-(A 3)$, (B1)-(B3) and $(C 1)-(C 3)$ hold. Then for each $\varphi \in \mathscr{C}\left(\mathcal{H}^{\alpha}\right)$, there exists a unique global mild solution to (3.1) with cylindrical $\mathcal{U}$-valued $F B M B_{Q}^{H}$ or Brownian motion $B_{Q}$ instead of $B_{Q}^{\sigma, \lambda}$.

In particular, as we will analyze in Section 5 the long time behavior of our model in the particular case of additive noise, i.e. when we replace $g$ by $\phi:[0, \infty) \mapsto \mathcal{L}_{Q}^{0}(\mathcal{U}, \mathcal{H})$ in $(3.1)$, we will state now how the previous results read in this case. For $\phi:[0, \infty) \mapsto \mathcal{L}_{Q}^{0}(\mathcal{U}, \mathcal{H})$ we assume the following condition:

(D1) There exists a constant $p \in\left(\frac{1}{1-2 \alpha}, \infty\right)$ such that

$$
\int_{0}^{\infty}\|\phi(r)\|_{Q}^{2 p} d r:=K<\infty
$$

By modifying slightly the proofs of theorems 1 and 2, we have

Corollary 2 Let $0<\alpha<\frac{1}{2}$ and assume that assumptions $(A 1)-(A 3)$ and $(B 1)-(B 3)$ hold true. If $\phi$ : $[0, \infty) \mapsto \mathcal{L}_{Q}^{0}(\mathcal{U}, \mathcal{H})$ satisfies $(D 1)$, then for each $\varphi \in \mathscr{C}\left(\mathcal{H}^{\alpha}\right)$, there exists a unique global mild solution to (3.1) but with $g$ replaced by $\phi$.

Similar to Corollary 1, we have

Corollary 3 Let $0<\alpha<\frac{1}{2}$ and assume that assumptions (A1)-(A3), (B1)-(B3) and (D1) hold. Then for each $\varphi \in \mathscr{C}\left(\mathcal{H}^{\alpha}\right)$, there exists a unique global mild solution to (3.1) with $\phi$ instead of $g$ and cylindrical $\mathcal{U}$-valued FBM $B_{Q}^{H}$ or Brownian motion $B_{Q}$ instead of $B_{Q}^{\sigma, \lambda}$.

Remark 1 Notice that our results concerning infinite delays can easily cover the case of bounded ones. More precisely, in the case of bounded delay, we consider the Banach space $C\left(-r, 0 ; L^{2}\left(\Omega ; \mathcal{H}^{\alpha}\right)\right)$ with the norm

$$
\|\psi\|_{C\left(-r, 0 ; L^{2}\left(\Omega ; \mathcal{H}^{\alpha}\right)\right)}=\left(\sup _{\theta \in[-r, 0]} E\|\psi(\theta)\|_{\alpha}^{2}\right)^{\frac{1}{2}}, \quad \psi \in C\left(-r, 0 ; L^{2}\left(\Omega ; \mathcal{H}^{\alpha}\right)\right)
$$

where $r$ is a fixed number. Then we replace $\mathscr{C}\left(\mathcal{H}^{\alpha}\right)$ by $C\left(-r, 0 ; L^{2}\left(\Omega ; \mathcal{H}^{\alpha}\right)\right)$, and by a similar argument as above, the existence and uniqueness of global mild solutions to (3.1) also hold true for bounded delay case.

Now we present an example to illustrate the type of delays that can be considered in our framework. Namely, we will consider two functions $f$ and $g$ containing a distributed delay and a variable delay, respectively.

Let $\mathcal{O}$ be a bounded open domain in $\mathbb{R}^{n}$ with smooth boundary $\partial \mathcal{O}$. Let $\mathcal{U}=\mathcal{H}=L^{2}(\mathcal{O})$, and let $A=-\Delta$ on the domain $\mathcal{O}$ with Dirichlet boundary condition.

Let $F:[0, \infty) \times(-\infty, 0] \times \mathcal{O} \mapsto \mathcal{O}$ and $G:[0, \infty) \times L^{2}(\mathcal{O}) \mapsto \mathcal{L}\left(L^{2}(\mathcal{O}), L^{2}(\mathcal{O})\right)$ be measurable functions satisfying the following assumptions:

(a) There exists a function $\mathcal{L}_{1}:(-\infty, 0] \mapsto[0, \infty)$ such that

$$
|F(t, s, u)-F(t, s, w)| \leq \mathcal{L}_{1}(s)|u-w|, \quad \forall t \geq 0, s \leq 0, u, w \in \mathcal{O}
$$


where $|\cdot|$ denotes the norm of $\mathbb{R}^{n}$ and $\int_{-\infty}^{0} \mathcal{L}_{1}(s) d s<\infty$.

(b) There exists a function $\mathcal{L}_{2}:(-\infty, 0] \mapsto[0, \infty)$ such that

$$
|F(t, s, v)| \leq \mathcal{L}_{2}(s)(1+|v|), \quad \forall t \geq 0, s \leq 0, v \in \mathcal{O}
$$

where $\int_{-\infty}^{0} \mathcal{L}_{2}(s) d s<\infty$.

(c) There exists a constant $\mathcal{K}_{1}>0$ such that

$$
\|G(t, v)-G(t, w)\|_{\mathcal{L}\left(L^{2}(\mathcal{O}), L^{2}(\mathcal{O})\right)} \leq \mathcal{K}_{1}\|v-w\|, \quad \forall t \geq 0, v, w \in L^{2}(\mathcal{O})
$$

where $\|\cdot\|_{\mathcal{L}\left(L^{2}(\mathcal{O}), L^{2}(\mathcal{O})\right)}$ denotes the norm of $\mathcal{L}\left(L^{2}(\mathcal{O}), L^{2}(\mathcal{O})\right)$.

(d) There exist a nonnegative function $\mathcal{K}_{2} \in L^{p}\left(\mathbb{R}^{+}\right)$with $p \in\left(\frac{1}{1-2 \alpha}, \infty\right)$ and a constant $\mathcal{K}_{3}>0$ such that

$$
\|G(t, v)\| \leq \mathcal{K}_{2}(t)+\mathcal{K}_{3}\|v\|, \quad \forall t \geq 0, v \in \mathcal{L}^{2}(\mathcal{O})
$$

Then we define

$$
f(t, \xi)(x):=\int_{-\infty}^{0} F(t, s, \xi(s)(x)) d s
$$

and

$$
g(t, \xi):=G(t, \xi(-\rho(t)))
$$

with $\rho \in C(\mathbb{R} ;[0, \infty))$, for each $t \in[0, \infty), \xi \in \mathscr{C}\left(\mathcal{H}^{\alpha}\right)$ and $x \in \mathcal{O}$. In this case, the delay terms $f$ and $g$ in (3.1) become

$$
f\left(t, u_{t}\right):=\int_{-\infty}^{0} F(t, s, u(t+s)) d s
$$

and

$$
g\left(t, u_{t}\right):=G(t, u(t-\rho(t)))
$$

In the sequel $C$ denotes an arbitrary positive constant, which may be different from line to line and even in the same line.

For any $\xi, \eta \in \mathscr{C}\left(\mathcal{H}^{\alpha}\right)$, by conditions $(a)$ and $(b)$, we obtain

$$
\begin{aligned}
E\|f(t, \xi)-f(t, \eta)\|^{2} & =E\left\|\int_{-\infty}^{0}(F(t, s, \xi(s))-F(t, s, \eta(s))) d s\right\|^{2} \\
& \leq E\left\|\int_{-\infty}^{0} \mathcal{L}_{1}(s)|\xi(s)-\eta(s)| d s\right\|^{2} \\
& \leq \int_{-\infty}^{0} \mathcal{L}_{1}(s) d s \int_{-\infty}^{0} \mathcal{L}_{1}(s) E\|\xi(s)-\eta(s)\|^{2} d s \\
& \leq\left(\int_{-\infty}^{0} \mathcal{L}_{1}(s) d s\right)^{2}\|\xi-\eta\|_{\mathscr{C}\left(\mathcal{H}^{\alpha}\right)}^{2}
\end{aligned}
$$


and

$$
\begin{aligned}
E\|f(t, \xi)\|^{2} & =E\left\|\int_{-\infty}^{0} F(t, s, \xi(s)) d s\right\|^{2} \\
& \leq E\left\|\int_{-\infty}^{0} \mathcal{L}_{2}(s)(1+|\xi(s)|) d s\right\|^{2} \\
& \leq C\left(\int_{-\infty}^{0} \mathcal{L}_{2}(s) d s\right)^{2}+C \int_{-\infty}^{0} \mathcal{L}_{2}(s) d s \int_{-\infty}^{0} \mathcal{L}_{2}(s) E\|\xi(s)\|^{2} d s \\
& \leq C+C\|\xi\|_{\mathscr{C}\left(\mathcal{H}^{\alpha}\right)}^{2} .
\end{aligned}
$$

Hence, $f$ satisfies $(B 1)-(B 3)$.

For $g$, by using conditions $(c)$ and $(d)$, we have

$$
\begin{aligned}
E\|g(t, \xi)-g(t, \eta)\|_{Q}^{2} & =E\|G(t, \xi(-\rho(t)))-G(t, \eta(-\rho(t)))\|_{Q}^{2} \\
& \leq C E\|G(t, \xi(-\rho(t)))-G(t, \eta(-\rho(t)))\|_{\mathcal{L}\left(L^{2}(\mathcal{O}), L^{2}(\mathcal{O})\right)}^{2} \\
& \leq C E\|\xi(-\rho(t))-\eta(-\rho(t))\|^{2} \\
& \leq C\|\xi-\eta\|_{\mathscr{C}\left(\mathcal{H}^{\alpha}\right)}^{2}
\end{aligned}
$$

and

$$
\begin{aligned}
E\|g(t, \xi)\|_{Q}^{2} & =E\|G(t, \xi(-\rho(t)))\|_{Q}^{2} \\
& \leq C E\|G(t, \xi(-\rho(t)))\|_{\mathcal{L}\left(L^{2}(\mathcal{O}), L^{2}(\mathcal{O})\right)}^{2} \\
& \leq C E\left(\mathcal{K}_{2}(t)+\mathcal{K}_{3}\|\xi(-\rho(t))\|\right)^{2} \\
& \leq C\left(\mathcal{K}_{2}(t)\right)^{2}+C\|\xi\|_{\mathscr{C}\left(\mathcal{H}^{\alpha}\right)}^{2},
\end{aligned}
$$

where $\|\cdot\|_{Q}$ denotes the norm of $\mathcal{L}_{Q}^{0}\left(L^{2}(\mathcal{O}), L^{2}(\mathcal{O})\right)$. Then $(C 1)-(C 3)$ hold true for $g$.

\section{The effect of noise on SPDEs with delay}

In this section we consider the effect of nonlinear noise on the following stochastic evolution equation with infinite delay:

$$
\left\{\begin{array}{l}
d u(t)=-A u(t) d t+\eta g\left(t, u_{t}\right) d B_{Q}^{\sigma, \lambda}(t), \quad t>0 \\
u(t)=\varphi(t), \quad t \in(-\infty, 0]
\end{array}\right.
$$

where $A, \varphi$ and $B_{Q}^{\sigma, \lambda}$ are as in problem (3.1), $g$ satisfies conditions $(C 1)-(C 2)$ and $g(t, 0)=0$ for any $t>0$, and the number $\eta$ is a positive parameter; this is the so-called level of the noise.

The following theorem shows that the upper excitation index of the solution $u$ of (4.1) at time $t$ is less than $2 \widetilde{p}$. 
Theorem 3 Let $0<\alpha<\frac{1}{2}$. Suppose that assumptions $(A 1)-(A 3),(C 1)-(C 2)$ and $g(t, 0)=0$ for any $t>0$ hold. Then there exists a constant $\widetilde{p} \in\left(\frac{1}{1-2 \alpha}, \infty\right)$ such that for each $\varphi \in \mathscr{C}\left(\mathcal{H}^{\alpha}\right)$,

$$
\limsup _{\eta \rightarrow \infty} \frac{\log \log \left\|u_{t}\right\|_{\mathscr{C}\left(\mathcal{H}^{\alpha}\right)}}{\log \eta} \leq 2 \widetilde{p},
$$

where $u(\cdot)$ denotes the solution of $(4.1)$.

Proof Firstly, observe that $(C 2)$ and $g(t, 0)=0(\forall t>0)$ ensure that

$$
E\|g(t, \xi)\|_{Q}^{2} \leq k_{1}(t)\|\xi\|_{\mathscr{C}\left(\mathcal{H}^{\alpha}\right)}^{2} .
$$

Combining this with $(A 1)-(A 2)$, we deduce from Lemma 2 and Hölder's inequality that

$$
\begin{aligned}
& E\|u(t)\|_{\alpha}^{2} \leq 2 E\|S(t) \varphi(0)\|_{\alpha}^{2}+2 \eta^{2} E\left\|\int_{0}^{t} S(t-r) g\left(r, u_{r}\right) d B_{Q}^{\sigma, \lambda}(r)\right\|_{\alpha}^{2} \\
& \leq 2 G^{2} e^{-2 \delta t} E\|\varphi(0)\|_{\alpha}^{2}+2 \eta^{2} G_{\alpha}^{2} N_{t} \int_{0}^{t} e^{-2 \delta(t-r)}(t-r)^{-2 \alpha} E\left\|g\left(r, u_{r}\right)\right\|_{Q}^{2} d r \\
& \leq 2 G^{2} e^{-2 \delta t} E\|\varphi(0)\|_{\alpha}^{2}+2 \eta^{2} G_{\alpha}^{2} N_{t} K_{1}\left(\int_{0}^{t} e^{-2 \delta \widetilde{q}(t-r)}(t-r)^{-2 \alpha \widetilde{q}} d r\right)^{\frac{1}{\widetilde{q}}}\left(\int_{0}^{t}\left\|u_{r}\right\|_{\mathscr{C}\left(\mathcal{H}^{\alpha}\right)}^{2 \widetilde{p}} d r\right)^{\frac{1}{\widetilde{p}}} \\
& \leq 2 G^{2} e^{-2 \delta t} E\|\varphi(0)\|_{\alpha}^{2}+2 \eta^{2} G_{\alpha}^{2} N_{t} K_{1}(2 \delta \widetilde{q})^{\frac{2 \alpha \widetilde{q}-1}{\tilde{q}}}(\Gamma(1-2 \alpha \widetilde{q}))^{\frac{1}{\widetilde{q}}}\left(\int_{0}^{t}\left\|u_{r}\right\|_{\mathscr{C}\left(\mathcal{H}^{\alpha}\right)}^{2 \widetilde{p}} d r\right)^{\frac{1}{\tilde{p}}}
\end{aligned}
$$

where $N_{t}$ is given in (3.10) and $\frac{1}{\widetilde{p}}+\frac{1}{\widetilde{q}}=1$. Replacing $t$ by $t+\theta$ in (4.4), we obtain that

$$
\begin{aligned}
& \sup _{\theta \in[-t, 0]} E\|u(t+\theta)\|_{\alpha}^{2} \leq 2 G^{2} E\|\varphi(0)\|_{\alpha}^{2}+2 \eta^{2} G_{\alpha}^{2} N_{t} K_{1}(2 \delta \widetilde{q})^{\frac{2 \alpha \widetilde{q}-1}{\tilde{q}}} \\
& \times(\Gamma(1-2 \alpha \widetilde{q}))^{\frac{1}{\tilde{q}}}\left(\int_{0}^{t}\left\|u_{r}\right\|_{\mathscr{C}\left(\mathcal{H}^{\alpha}\right)}^{2 \widetilde{\widetilde{q}}} d r\right)^{\frac{1}{\tilde{P}}}
\end{aligned}
$$

Therefore,

$$
\begin{aligned}
\left\|u_{t}\right\|_{\mathscr{C}\left(\mathcal{H}^{\alpha}\right)}^{2} \leq\left(2 G^{2}+1\right)\|\varphi\|_{\mathscr{C}\left(\mathcal{H}^{\alpha}\right)}^{2} & +2 \eta^{2} G_{\alpha}^{2} N_{t} K_{1}(2 \delta \widetilde{q})^{\frac{2 \alpha \tilde{q}-1}{\tilde{q}}} \\
& \times(\Gamma(1-2 \alpha \widetilde{q}))^{\frac{1}{\tilde{q}}}\left(\int_{0}^{t}\left\|u_{r}\right\|_{\mathscr{C}\left(\mathcal{H}^{\alpha}\right)}^{2 \widetilde{p}} d r\right)^{\frac{1}{\widetilde{p}}},
\end{aligned}
$$

and consequently,

$$
\begin{aligned}
& \left\|u_{t}\right\|_{\mathscr{C}\left(\mathcal{H}^{\alpha}\right)}^{2 \widetilde{p}} \leq 2^{\widetilde{p}-1}\left(2 G^{2}+1\right)^{\widetilde{p}}\|\varphi\|_{\mathscr{C}\left(\mathcal{H}^{\alpha}\right)}^{2 \widetilde{p}} \\
& \quad+2^{2 \widetilde{p}-1} \eta^{2 \widetilde{p}} G_{\alpha}^{2 \widetilde{p}} N_{t}^{\widetilde{p}} K_{1}^{\widetilde{p}}(2 \delta \widetilde{q})^{\frac{(2 \alpha \widetilde{q}-1) \widetilde{q}}{\tilde{q}}}(\Gamma(1-2 \alpha \widetilde{q}))^{\frac{\widetilde{p}}{\tilde{q}}} \int_{0}^{t}\left\|u_{r}\right\|_{\mathscr{C}\left(\mathcal{H}^{\alpha}\right)}^{2 \widetilde{p}} d r .
\end{aligned}
$$


Gronwall's lemma conduces us to

$$
\left\|u_{t}\right\|_{\mathscr{C}\left(\mathcal{H}^{\alpha}\right)}^{2 \widetilde{p}} \leq \Pi_{5} e^{\Pi_{6} \eta^{2 \widetilde{p}} N_{t}^{\widetilde{p}} t}
$$

where we have used the notations

$$
\Pi_{5}:=2^{\widetilde{p}-1}\left(2 G^{2}+1\right)^{\widetilde{p}}\|\varphi\|_{\mathscr{C}\left(\mathcal{H}^{\alpha}\right)}^{2 \widetilde{p}}
$$

and

$$
\Pi_{6}:=2^{2 \widetilde{p}-1} G_{\alpha}^{2 \widetilde{p}} K_{1}^{\widetilde{p}}(2 \delta \widetilde{q})^{\frac{(2 \alpha \widetilde{q}-1) \widetilde{p}}{\widetilde{q}}}(\Gamma(1-2 \alpha \widetilde{q}))^{\frac{\widetilde{p}}{\tilde{q}}}
$$

The conclusion (4.2) follows immediately from (4.6), and thus the proof is complete.

Remark 2 In particular, let $\alpha=0$ in Theorem 3, then for each $\varphi \in \mathscr{C}(\mathcal{H})$ the unique mild solution $u$ to $(4.1)$ satisfies

$$
\limsup _{\eta \rightarrow \infty} \frac{\log \log \left\|u_{t}\right\|_{\mathscr{C}(\mathcal{H})}}{\log \eta} \leq 2
$$

where

$$
\|\psi\|_{\mathscr{C}(\mathcal{H})}=\left(\sup _{\theta \in(-\infty, 0]} E\|\psi(\theta)\|^{2}\right)^{\frac{1}{2}}, \quad \psi \in \mathscr{C}(\mathcal{H})
$$

As a simple consequence of Theorem 3, in view of Lemma 3, we obtain

Corollary 4 Let $0<\alpha<\frac{1}{2}$ and assume that assumptions $(A 1)-(A 3),(C 1)-(C 2)$ and $g(0)=0$ hold. Then there exists a constant $p^{\prime} \in\left(\frac{1}{1-2 \alpha}, \infty\right)$ such that for each $\varphi \in \mathscr{C}\left(\mathcal{H}^{\alpha}\right)$, the unique mild solution $u$ to (4.1) with cylindrical $\mathcal{U}$-valued $F B M B_{Q}^{H}$ or Brownian motion $B_{Q}$ instead of $B_{Q}^{\sigma, \lambda}$ satisfies

$$
\limsup _{\eta \rightarrow \infty} \frac{\log \log \left\|u_{t}\right\|_{\mathscr{C}\left(\mathcal{H}^{\alpha}\right)}}{\log \eta} \leq 2 p^{\prime}
$$

Remark 3 If we replace $\mathscr{C}\left(\mathcal{H}^{\alpha}\right)$ by $C\left(-r, 0 ; L^{2}\left(\Omega ; \mathcal{H}^{\alpha}\right)\right)$, then the results in this section also hold true for bounded delay case.

\section{Exponential decay of solutions in mean square}

In this section we are interested in the exponential decay to zero in mean square of the mild solutions.

Observe that in Lemmas 2 and 3, the right hand side of inequalities for the stochastic integrals with respect to TFBM and FBM, respectively, are

$$
(2 H-1) t^{2 H-1} \beta\left(2-2 H, H-\frac{1}{2}\right)+4 \lambda^{2} t^{2 H+1} \frac{\beta\left(2-2 H, H+\frac{1}{2}\right)}{2 H-1}
$$

and

$$
2 H t^{2 H-1} \text {. }
$$


Comparing with the stochastic integral with respect to Brownian motion, (5.1) and (5.2) are dependent on $t$ and tend to infinity as $t \rightarrow \infty$. It is difficult to prove that the mild solutions to problem (3.1) with cylindrical $\mathcal{U}$-valued TFBM $B_{Q}^{\sigma, \lambda}$ or FBM $B_{Q}^{H}$ exponentially decay to zero in mean square. Hence in this section we consider the following stochastic evolution equation with infinite delay:

$$
\left\{\begin{array}{l}
d u(t)=-A u(t) d t+f\left(t, u_{t}\right) d t+\phi(t) d B_{Q}^{\sigma, \lambda}(t), \quad t>0 \\
u(t)=\varphi(t), \quad t \in(-\infty, 0]
\end{array}\right.
$$

where $A$ and $B_{Q}^{\sigma, \lambda}$ are as in problem (3.1).

On the other hand, if we still consider the space $\mathscr{C}\left(\mathcal{H}^{\alpha}\right)$ given in Section 3, then we need to replace $t$ by $t+\theta$ in (5.10) and take the sup norm $\sup _{\theta \in[-t, 0]} E\|u(t+\theta)\|_{\alpha}^{2}$, but the exponential decay terms $e^{-2 \delta t}$ and $e^{-\delta t}$ disappear when we take the sup norm. In this case, we cannot obtain the exponential decay property for the mild solutions. However, this problem can be overcome if we use another space $\mathscr{C}^{\gamma}\left(\mathcal{H}^{\alpha}\right)$ given later, which was extensively applied to investigate infinite delay case, see, e.g., $[5,26]$ and the references therein. It is worth mentioning that considering this new space $\mathscr{C}^{\gamma}\left(\mathcal{H}^{\alpha}\right)$ will allow us to prove exponential decay of solutions, but will restrict the type of unbounded delay terms which can appear in the function $f$, for instance, general variable delay terms cannot be considered, but with our current space $\mathscr{C}\left(\mathcal{H}^{\alpha}\right)$ we can include both variable and distributed infinite unbounded delays but, in general, we may not be able to prove exponential decay of solutions, as it is shown in [21] for the case of stochastic 2D-Navier Stokes with infinite delay.

We define the abstract phase space $\mathscr{C}^{\gamma}\left(\mathcal{H}^{\alpha}\right)$ by

$$
\mathscr{C}^{\gamma}\left(\mathcal{H}^{\alpha}\right)=\left\{\psi \in C\left(-\infty, 0 ; L^{2}\left(\Omega ; \mathcal{H}^{\alpha}\right)\right): \lim _{\theta \rightarrow-\infty} e^{\gamma \theta} E\|\psi(\theta)\|_{\alpha}^{2} \text { exists }\right\},
$$

where the parameter $\gamma>0$. If $\mathscr{C}^{\gamma}\left(\mathcal{H}^{\alpha}\right)$ is endowed with the norm

$$
\|\psi\|_{\mathscr{C}^{\gamma}\left(\mathcal{H}^{\alpha}\right)}=\left(\sup _{\theta \in(-\infty, 0]} e^{\gamma \theta} E\|\psi(\theta)\|_{\alpha}^{2}\right)^{\frac{1}{2}}, \quad \psi \in \mathscr{C}^{\gamma}\left(\mathcal{H}^{\alpha}\right)
$$

then $\left(\mathscr{C}^{\gamma}\left(\mathcal{H}^{\alpha}\right),\|\cdot\|_{\mathscr{C}^{\gamma}\left(\mathcal{H}^{\alpha}\right)}\right)$ is a Banach space.

We now need to state the following conditions:

$(B 1)^{\prime}$ For any $\xi \in \mathscr{C}^{\gamma}\left(\mathcal{H}^{\alpha}\right)$, the mapping $[0, \infty) \ni t \mapsto f(t, \xi) \in \mathcal{H}$ is measurable.

$(B 2)^{\prime}$ There exists a nonnegative function $l_{4} \in L^{\infty}\left(\mathbb{R}^{+}\right)$such that for any $\xi, \eta \in \mathscr{C}^{\gamma}\left(\mathcal{H}^{\alpha}\right)$ and $t \geq 0$,

$$
E\|f(t, \xi)-f(t, \eta)\|^{2} \leq l_{4}(t)\|\xi-\eta\|_{\mathscr{C}^{\gamma}\left(\mathcal{H}^{\alpha}\right)}^{2}
$$

and $\left\|l_{4}\right\|_{L^{\infty}\left(\mathbb{R}^{+}\right)}:=L_{4}<\infty$.

$(B 3)^{\prime}$ There exist nonnegative functions $l_{5} \in L^{1}\left(\mathbb{R}^{+}\right)$and $l_{6} \in L^{\infty}\left(\mathbb{R}^{+}\right)$such that for any $\xi \in \mathscr{C}^{\gamma}\left(\mathcal{H}^{\alpha}\right)$ and $t \geq 0$,

$$
E\|f(t, \xi)\|^{2} \leq l_{5}(t)+l_{6}(t)\|\xi\|_{\mathscr{C}^{\gamma}\left(\mathcal{H}^{\alpha}\right)}^{2},
$$


and

$$
\int_{0}^{\infty} e^{\delta r} l_{5}(r) d r:=L_{5}<\infty, \quad\left\|l_{6}\right\|_{L^{\infty}\left(\mathbb{R}^{+}\right)}:=L_{6}<\infty .
$$

$(C 1)^{\prime}$ There exists a constant $p \in\left(\frac{1}{1-2 \alpha}, \infty\right)$ such that

$$
\int_{0}^{\infty} e^{\delta p r}\|\phi(r)\|_{Q}^{2 p} d r:=\Lambda<\infty
$$

Theorem 4 Let $0<\alpha<\frac{1}{2}$. Assume that the assumptions $(A 1)-(A 3),(B 1)^{\prime}-(B 3)^{\prime},(C 1)^{\prime}$ and

$$
\gamma>2 \delta>2 \Pi_{9}
$$

hold, where $\Pi_{9}:=3 G_{\alpha}^{2} \delta^{2 \alpha-1} \Gamma(1-2 \alpha) L_{6}$. Then, there exists a constant $a>0$ such that for any mild solution $u$ of (5.3) with the initial condition $\varphi \in \mathscr{C}^{\gamma}\left(\mathcal{H}^{\alpha}\right)$,

$$
\limsup _{t \rightarrow \infty}\left(\frac{1}{t}\right) \log \left\|u_{t}\right\|_{\mathscr{C} \gamma\left(\mathcal{H}^{\alpha}\right)}^{2} \leq-a
$$

Proof Thanks to (3.2), we have

$$
\begin{aligned}
E\|u(t)\|_{\alpha}^{2} \leq & 3 E\|S(t) \varphi(0)\|_{\alpha}^{2}+3 E\left\|\int_{0}^{t} S(t-r) f\left(r, u_{r}\right) d r\right\|_{\alpha}^{2} \\
& +3 E\left\|\int_{0}^{t} S(t-r) \phi(r) d B_{Q}^{\sigma, \lambda}(r)\right\|_{\alpha}^{2}:=I_{9}+I_{10}+I_{11} .
\end{aligned}
$$

By condition $(A 1)$, we obtain

$$
I_{9} \leq 3 G^{2} e^{-2 \delta t} E\|\varphi(0)\|_{\alpha}^{2} .
$$

For $I_{10}$, by conditions $(A 2),(B 3)^{\prime}$ and Hölder's inequality, we deduce that

$$
\begin{aligned}
I_{10} & \leq 3 G_{\alpha}^{2} E\left(\int_{0}^{t} e^{-\delta(t-r)}(t-r)^{-\alpha}\left\|f\left(r, u_{r}\right)\right\| d r\right)^{2} \\
& \leq 3 G_{\alpha}^{2} \int_{0}^{t} e^{-\delta(t-r)}(t-r)^{-2 \alpha} d r \int_{0}^{t} e^{-\delta(t-r)} E\left\|f\left(r, u_{r}\right)\right\|^{2} d r \\
& \leq 3 G_{\alpha}^{2} \delta^{2 \alpha-1} \Gamma(1-2 \alpha) \int_{0}^{t} e^{-\delta(t-r)}\left(l_{5}(r)+l_{6}(r)\left\|u_{r}\right\|_{\mathscr{C}^{\gamma}\left(\mathcal{H}^{\alpha}\right)}^{2}\right) d r \\
& \leq 3 G_{\alpha}^{2} \delta^{2 \alpha-1} \Gamma(1-2 \alpha) e^{-\delta t} L_{5}+3 G_{\alpha}^{2} \delta^{2 \alpha-1} \Gamma(1-2 \alpha) e^{-\delta t} L_{6} \int_{0}^{t} e^{\delta r}\left\|u_{r}\right\|_{\mathscr{C}^{\gamma}\left(\mathcal{H}^{\alpha}\right)}^{2} d r
\end{aligned}
$$


For $I_{11}$, it follows from conditions $(A 2),(C 1)^{\prime}$, Hölder's inequality and Lemma 2 that

$$
\begin{aligned}
I_{11} & \leq 3 N_{t} G_{\alpha}^{2} \int_{0}^{t} e^{-2 \delta(t-r)}(t-r)^{-2 \alpha}\|\phi(r)\|_{Q}^{2} d r \\
& \leq 3 N_{t} G_{\alpha}^{2} e^{-\delta t}\left(\int_{0}^{t}(t-r)^{-2 \alpha q} e^{-\delta q(t-r)} d r\right)^{\frac{1}{q}}\left(\int_{0}^{t} e^{\delta p r}\|\phi(r)\|_{Q}^{2 p} d r\right)^{\frac{1}{p}} \\
& \leq 3 N_{t} G_{\alpha}^{2} e^{-\delta t}(\delta q)^{\frac{2 \alpha q-1}{q}}(\Gamma(1-2 \alpha q))^{\frac{1}{q}} \Lambda^{\frac{1}{p}},
\end{aligned}
$$

where $q$ and $N_{t}$ are given in (3.10). Therefore,

$$
\begin{aligned}
E\|u(t)\|_{\alpha}^{2} \leq 3 & G^{2} e^{-2 \delta t} E\|\varphi(0)\|_{\alpha}^{2}+3 G_{\alpha}^{2} \delta^{2 \alpha-1} \Gamma(1-2 \alpha) e^{-\delta t} L_{5} \\
& +3 G_{\alpha}^{2} \delta^{2 \alpha-1} \Gamma(1-2 \alpha) e^{-\delta t} L_{6} \int_{0}^{t} e^{\delta r}\left\|u_{r}\right\|_{\mathscr{C}^{\gamma}\left(\mathcal{H}^{\alpha}\right)}^{2} d r \\
& +3 N_{t} G_{\alpha}^{2} e^{-\delta t}(\delta q)^{\frac{2 \alpha q-1}{q}}(\Gamma(1-2 \alpha q))^{\frac{1}{q}} \Lambda^{\frac{1}{p}}
\end{aligned}
$$

By assumption (5.4), we have $e^{(\gamma-2 \delta) \theta} \leq 1$ for $\theta \leq 0$. Multiplying (5.10) by $e^{\gamma \theta} e^{-\gamma \theta}$ and replacing $t$ by $t+\theta$, we obtain that

$$
\begin{aligned}
\sup _{\theta \in[-t, 0]} e^{\gamma \theta} E\|u(t+\theta)\|_{\alpha}^{2} \leq 3 & G^{2} e^{-2 \delta t} E\|\varphi(0)\|_{\alpha}^{2}+3 G_{\alpha}^{2} \delta^{2 \alpha-1} \Gamma(1-2 \alpha) e^{-\delta t} L_{5} \\
& +3 G_{\alpha}^{2} \delta^{2 \alpha-1} \Gamma(1-2 \alpha) e^{-\delta t} L_{6} \int_{0}^{t} e^{\delta r}\left\|u_{r}\right\|_{\mathscr{C}^{\gamma}\left(\mathcal{H}^{\alpha}\right)}^{2} d r \\
& +3 N_{t} G_{\alpha}^{2} e^{-\delta t}(\delta q)^{\frac{2 \alpha q-1}{q}}(\Gamma(1-2 \alpha q))^{\frac{1}{q}} \Lambda^{\frac{1}{p}} .
\end{aligned}
$$

Note that $\gamma>2 \delta$, hence for all $\theta \in(-\infty,-t]$,

$$
e^{\gamma \theta} E\|u(t+\theta)\|_{\alpha}^{2} \leq e^{-\gamma t} e^{\gamma(t+\theta)} E\|\varphi(t+\theta)\|_{\alpha}^{2} \leq e^{-\gamma t}\|\varphi\|_{\mathscr{C}^{\gamma}\left(\mathcal{H}^{\alpha}\right)}^{2} \leq e^{-2 \delta t}\|\varphi\|_{\mathscr{C}^{\gamma}\left(\mathcal{H}^{\alpha}\right)}^{2} .
$$

(5.11) and (5.12) imply that

$$
e^{\delta t}\left\|u_{t}\right\|_{\mathscr{C} \gamma\left(\mathcal{H}^{\alpha}\right)}^{2} \leq \Pi_{7}+\Pi_{8} N_{t}+\Pi_{9} \int_{0}^{t} e^{\delta r}\left\|u_{r}\right\|_{\mathscr{C}^{\gamma}\left(\mathcal{H}^{\alpha}\right)}^{2} d r
$$

where we have used the notations

$$
\begin{aligned}
\Pi_{7} & :=\left(3 G^{2}+1\right)\|\varphi\|_{\mathscr{C}^{\gamma}\left(\mathcal{H}^{\alpha}\right)}^{2}+3 G_{\alpha}^{2} \delta^{2 \alpha-1} \Gamma(1-2 \alpha) L_{5}, \\
\Pi_{8} & :=3 G_{\alpha}^{2}(\delta q)^{\frac{2 \alpha q-1}{q}}(\Gamma(1-2 \alpha q))^{\frac{1}{q}} \Lambda^{\frac{1}{p}},
\end{aligned}
$$

and

$$
\Pi_{9}:=3 G_{\alpha}^{2} \delta^{2 \alpha-1} \Gamma(1-2 \alpha) L_{6}
$$


Applying Gronwall's lemma to (5.13), we have

$$
\left\|u_{t}\right\|_{\mathscr{C}^{\gamma}\left(\mathcal{H}^{\alpha}\right)}^{2} \leq\left(\Pi_{7}+\Pi_{8} N_{t}\right) e^{\left(\Pi_{9}-\delta\right) t}=\left(\Pi_{7}+\Pi_{8} N_{t}\right) e^{-a t}
$$

where $a=\delta-\Pi_{9}$. The proof is therefore complete.

Corollary 5 Let $0<\alpha<\frac{1}{2}$. Assume that assumptions $(A 1)-(A 3),(B 1)^{\prime}-(B 3)^{\prime},(C 1)^{\prime}$ and (5.4) hold. Then, there exists a constant $a^{\prime}>0$ such that for any mild solution $u$ of (5.3) with cylindrical $\mathcal{U}$-valued $F B M B_{Q}^{H}$ or Brownian motion $B_{Q}$ instead of $B_{Q}^{\sigma, \lambda}$ and the initial condition $\varphi \in \mathscr{C}^{\gamma}\left(\mathcal{H}^{\alpha}\right)$,

$$
\limsup _{t \rightarrow \infty}\left(\frac{1}{t}\right) \log \left\|u_{t}\right\|_{\mathscr{C}^{\gamma}\left(\mathcal{H}^{\alpha}\right)}^{2} \leq-a^{\prime}
$$

Remark 4 If we consider $C\left(-r, 0 ; L^{2}\left(\Omega ; \mathcal{H}^{\alpha}\right)\right)$ instead of $\mathscr{C}^{\gamma}\left(\mathcal{H}^{\alpha}\right)$ in this section, then by slightly modifying the proofs in Theorem 4 and Corollary 5 , we can obtain the exponential decay property of the mild solutions to (5.3) in the bounded delay case.

\section{References}

1. B. Blümich, White noise nonlinear system analysis in nuclear magnetic resonance spectroscopy, Prog. Nucl. Magn. Reson. Spectrosc. 19 (4) (1987) 331-417.

2. B. Boufoussi, S. Hajji, Neutral stochastic functional differential equations driven by a fractional Brownian motion in a Hilbert space, Statist. Probab. Lett. 82 (8) (2012) 1549-1558.

3. J. P. Pérez Beaupuits, A. Otárola, F. T. Rantakyrö, R. C. Rivera, S. J. E. Radford, L.-Å Nyman, Analysis of wind data gathered at Chajnantor, ALMA Memo 497 (2004).

4. R. F. Curtain, P. L. Falb, Stochastic differential equations in Hilbert space, J. Differ. Equ. 10 (3) (1971) 412-430.

5. T. Caraballo, M. J. Garrido-Atienza, B. Schmalfuss, J. Valero, Non-autonomous and random attractors for delay random semilinear equations without uniqueness, Discrete Contin. Dyn. Syst. 21 (2008) 415-443.

6. T. Caraballo, M. J. Garrido-Atienza, T. Taniguchi, The existence and exponential behavior of solutions to stochastic delay evolution equations with a fractional Brownian motion, Nonlinear Anal. 74 (11) (2011) 3671-3684.

7. T. Caraballo, M. A. Hammami, L. Mchiri, Practical exponential stability of impulsive stochastic functional differential equations, Systems Control Lett. 109 (2017) 43-48.

8. G. L. Chen, D. S. Li, L. Shi, O. van Gaans, S. V. Lunel, Stability results for stochastic delayed recurrent neural networks with discrete and distributed delays, J. Differ. Equ. 264 (6) (2018) 3864-3898.

9. Y. Chen, X. D. Wang, W. H. Deng, Tempered fractional Langevin-Brownian motion with inverse $\beta$-stable subordinator, J. Phys. A: Math. Theor. 51 (2018) 495001.

10. A. G. Davenport, The spectrum of horizontal gustiness near the ground in high winds, Q. J. R. Meteorol. Soc. 87 (1961) 194-211.

11. G. Da Prato, J. Zabczyk, Stochastic Equations in Infinite Dimensions, Encyclopedia of Mathematics and Its Applications, Cambridge Univ. Press, Cambridge, MA, 1992.

12. M. Foondun, M. Joseph, Remarks on non-linear noise excitability of some stochastic heat equations, Stoch. Process. Appl. 124 (10) (2014) 3429-3440.

13. M. Ferrante, C. Rovira, Stochastic delay differential equations driven by fractional Brownian motion with Hurst parameter $H>\frac{1}{2}$, Bernoulli 12 (2006) 85-100.

14. M. Ferrante, C. Rovira, Convergence of delay differential equations driven by fractional Brownian motion, J. Evol. Equ. 10 (4) (2010) 761-783. 
15. M. J. Garrido-Atienza, K. N. Lu, B. Schmalfuss, Local pathwise solutions to stochastic evolution equations driven by fractional Brownian motions with Hurst parameters $H \in(1 / 3,1 / 2]$, Discrete Contin. Dyn. Syst. Ser. B. 20 (8) (2015) 2553-2581.

16. M. J. Garrido-Atienza, K. N. Lu, B. Schmalfuss, Random dynamical systems for stochastic evolution equations driven by multiplicative fractional Brownian noise with Hurst parameters $H \in[1 / 3,1 / 2]$, SIAM J. Appl. Dyn. Syst. 15 (1) (2016) 625-654.

17. J. J. Jang, J. S. Guo, Analysis of maximum wind force for offshore structure design, J. Mar. Sci. Technol. 7 (1) (1999) 43-51.

18. P. T. Hong, C. T. Binh, A note on exponential stability of non-autonomous linear stochastic differential delay equations driven by a fractional Brownian motion with Hurst index $>\frac{1}{2}$, Statist. Probab. Lett. 138 (2018) 127-136.

19. D. Khoshnevisan, K. Kim, Nonlinear noise excitation of intermittent stochastic PDEs and the topology of LCA groups, Ann. Probab. 43 (4) (2015) 1944-1991.

20. D. Khoshnevisan, K. Kim, Non-linear noise excitation and intermittency under high disorder, Proc. Am. Math. Soc. 143 (9) (2015) 4073-4083.

21. L. F. Liu, T. Caraballo, Analysis of a Stochastic 2D-Navier-Stokes Model with Infinite Delay, J. Dyn. Differ. Equ. 31 (2019), no. 4, 2249-2274.

22. B. Lindner, J. Garcia-Ojalvo, A. Neiman, L. Schimansky-Geier, Effects of noise in excitable systems, Phys. Rep. 392 (6) (2004) 321-424.

23. Y. S. Li, A. Kareem, ARMA systems in wind engineering, Probab. Eng. Mech. 5 (2) (1990) 49-59.

24. E. H. Lakhel, A. Tlidi, Controllability of time-dependent neutral stochastic functional differential equations driven by a fractional Brownian motion, J. Nonlinear Sci. Appl. 11 (2018), 850-863.

25. W. Liu, K. H. Tian, M. Foondun, On some properties of a class of fractional stochastic heat equations, J. Theoret. Probab. 30 (4) (2017) 1310-1333.

26. Y. J. Li, Y. J. Wang, The existence and asymptotic behavior of solutions to fractional stochastic evolution equations with infinite delay, J. Differ. Equ. 266 (6) (2019) 3514-3558.

27. M. M. Meerschaert, F. Sabzikar, Tempered fractional Brownian motion, Stat. Probab. Lett. 83 (10) (2013) 2269-2275.

28. M. M. Meerschaert, F. Sabzikar, Stochastic integration for tempered fractional Brownian motion, Stoch. Process. Appl. 124 (7) (2014) 2363-2387.

29. D. J. Norton, Mobile offshore platform wind loads, in: Proc. 13th Offshore Techn. Conf., OTC 4123, 4 (1981) 77-88.

30. A. Neuenkirch, I. Nourdin, S. Tindel, Delay equations driven by rough paths, Electron. J. Probab. 13 (67) (2008) $2031-2068$.

31. I. Norros, E. Valkeila, J. Virtamo, An elementary approach to a Girsanov formula and other analytical results on fractional Brownian motions, Bernoulli 5 (4) (1999) 571-587.

32. T. Taniguchi, K. Liu, A. Truman, Existence, uniqueness, and asymptotic behavior of mild solutions to stochastic functional differential equations in Hilbert spaces, J. Differ. Equ. 181 (1) (2002) 72-91.

33. X. H. Wang, K. N. Lu, B. X. Wang, Exponential stability of non-autonomous stochastic delay lattice systems with multiplicative noise, J. Dyn. Differ. Equ. 28 (2016) 1309-1335.

34. L. P. Xua, J. W. Luo, Viability for stochastic functional differential equations in Hilbert spaces driven by fractional Brownian motion, Appl. Math. Comput. 341 (2019) 93-110. 